\title{
A randomized controlled trial to isolate the effects of fasting and energy restriction on weight loss and metabolic health in lean adults
}

Authors: Templeman Iain $^{1}$, Smith Harry Alex ${ }^{1}$, Chowdhury Enhad ${ }^{1}$, Chen Yung-Chih ${ }^{1,2}$, Carroll Harriet ${ }^{1,3}$, Johnson-Bonson Drusus ${ }^{1}$, Hengist Aaron ${ }^{1}$, Smith Rowan ${ }^{1}$, Creighton Jade ${ }^{1,4}$, Clayton David ${ }^{4}$, Varley $\operatorname{Ian}^{4}$, Karagounis Leonidas ${ }^{5,6}$, Wilhelmsen Andrew ${ }^{7}$, Tsintzas Kostas ${ }^{7}$, Reeves Sue ${ }^{8}$, Walhin Jean-Philippe ${ }^{1}$, Gonzalez Javier Thomas ${ }^{1}$, Thompson Dylan ${ }^{1}$ \& Betts James Alexander ${ }^{1 *}$

\section{Affiliations:}

${ }^{1}$ Centre for Nutrition, Exercise \& Metabolism, Department for Health, University of Bath, BA2 7AY, UK.

${ }^{2}$ Department of Physical Education, National Taiwan Normal University, Taipei City 106, Taiwan.

${ }^{3}$ Cardiovascular Research - Hypertension, Clinical Research Centre, Lund University, 22100 , Sweden.

${ }^{4}$ School of Science and Technology, Nottingham Trent University, Nottingham, NG11 8NS, UK.

${ }^{5}$ Nestlé Health Science, Translation Research, Avenue Nestlé 55, CH-1800 Vevey, Switzerland.

${ }^{6}$ Institute of Social and Preventive Medicine, University of Bern, 3012 Bern, Switzerland.

${ }^{7}$ MRC Versus Arthritis Centre for Musculoskeletal Ageing Research, School of Life Sciences, University of Nottingham, Nottingham, NG7 2UH, UK.

${ }^{8}$ Department of Life Sciences, University of Roehampton, London, SW15 4JD, UK.

*Corresponding author. Email: J.Betts@bath.ac.uk 


\section{Overline: METABOLISM}

\section{One Sentence Summary:}

Daily energy restriction more effectively reduces body fat content than alternate-day fasting, with no evidence of fasting-specific health benefits.

\section{Abstract:}

Intermittent fasting may impart metabolic benefits independent of energy balance by initiating fasting-mediated mechanisms. This randomized controlled trial examined 24-h fasting with $150 \%$ energy intake on alternate days for 3 weeks $(\mathbf{0 : 1 5 0} ; n=12)$. Control groups involved a matched degree of energy restriction applied continuously without fasting $(75 \%$ energy intake daily; 75:75; $n=12)$ or a matched pattern of fasting without net energy restriction (200\% energy intake on alternate days; $\mathbf{0 : 2 0 0} ; \mathrm{n}=12$ ). Primary outcomes were body composition, components of energy balance, and post-prandial metabolism. Daily energy restriction (75:75) reduced body mass $(-1.91 \pm 0.99 \mathrm{~kg})$ almost entirely due to fat loss $(-1.75 \pm 0.79 \mathrm{~kg})$. Restricting energy intake via fasting (0:150) also decreased body mass $(-1.60 \pm 1.06 \mathrm{~kg} ; p=0.46$ versus $75: 75)$ but with attenuated reductions in body fat $(-0.74 \pm 1.32 \mathrm{~kg} ; p=0.01$ versus $75: 75)$, whereas fasting without energy restriction $(\mathbf{0 : 2 0 0})$ did not significantly reduce either body mass $(-0.52 \pm 1.09 \mathrm{~kg} ; p \leq 0.04$ versus $75: 75 \& 0: 150)$ or fat mass $(-0.12 \pm 0.68 \mathrm{~kg} ; p \leq 0.05$ versus $75: 75 \& 0: 150)$. Post-prandial indices of cardiometabolic health and gut hormones, along with the expression of key genes in subcutaneous adipose tissue, were not statistically different between groups $(p>0.05)$. Alternateday fasting less effectively reduces body fat mass than a matched degree of daily energy restriction and without evidence of fasting-specific effects on metabolic regulation or cardiovascular health. 


\section{Introduction}

Intermittent fasting is an umbrella term for dietary regimens involving temporal restriction of daily feeding patterns, including a fast on some days each week (for example the “5:2" diet), for part of each day (time-restricted feeding) or for part or all of every second day (modified/complete alternate-day fasting; 1). The apparent popularity of these approaches suggests that many people find adoption and adherence comparatively easy, potentially because we as a species are well adapted to erratic food availability as opposed to an abundant food supply and thus permanently post-prandial (fed) and lipogenic state throughout waking hours (2, 3). As neatly described previously, all mammals exhibit evolutionarily conserved (albeit somewhat species-specific) adaptive responses to food deprivation (4). Specifically, fastingmediated mechanisms stimulate lipolysis and ketogenesis to support energy requirements, whilst regulating various cell signalling pathways to efficiently recycle the limited nutrients available (reduced glycolysis and protein synthesis, with increased autophagy; 4, 5, 6).

Studies in mice clearly demonstrate that metabolic health can be improved by restricting food availability to certain periods (independent of energy intake or weight loss), although such fasting deviates more profoundly from these animals' naturally continuous foraging pattern than from the schedule of regular but less frequent meals more typical of human societies (7). Recent reviews of human trials indicate that intermittent fasting can elicit weight loss and health gains but that these effects are generally equivalent to standard energy restriction without restricted feeding times (that is, without fasting), indicating that metabolic effects may be due to weight loss rather than fasting per se $(1,8-10)$. However, trials to date have generally involved substantial reductions in energy intake but without prescribing complete abstinence from macronutrients throughout 'fasting' days, and so may not initiate the aforementioned fasting- 
mediated mechanisms (11). Indeed, at least 12-14 consecutive hours of absolute nutritional withdrawal is required to elicit frank depletion of hepatic glycogen reserves and the consequent transition towards oxidation of endogenous lipid-derived substrates (fatty acids and ketone bodies; 12, 13), which are proposed to increase insulin sensitivity, improve cardiovascular health, and preserve muscle mass during weight loss $(4,14)$.

Based upon the above reasoning, it becomes understandable why the small minority of human trials that have reported positive health outcomes peculiar to intermittent fasting (beyond that explained by weight loss) are those same few in which the post-absorptive state has been regularly sustained via uninterrupted fasting of at least $16 \mathrm{~h}(1)$. For example, early timerestricted feeding (fasting from $1500 \mathrm{~h}$ daily) in men with pre-diabetes improved their insulin sensitivity within five weeks without losing weight, simply by extending their usual overnight fast to $18 \mathrm{~h}$ (15). This effect on insulin sensitivity has since been confirmed in healthy young adults after just 2 weeks of fasting for $16 \mathrm{~h}$ from $1600 \mathrm{~h}$ each day (16) and after 8 weeks of simply restricting typical daily meals to an 11-h period ending at $1900 \mathrm{~h}$ (17). The other common form of intermittent fasting that routinely involves such protracted post-absorptive periods is complete alternate-day fasting (no energy intake whatsoever during fasting days), yet almost no research has examined fasting periods that span an entire day. One recent trial has shown that strict $\sim 36 \mathrm{~h}$ fasting periods alternated with $\sim 12-\mathrm{h}$ ad libitum eating every other day can markedly reduce both fat mass and cardiovascular disease risk markers amongst non-obese adults within four weeks (18). However, that study did not include the continuous energy restriction control group necessary to dissociate the effects of fasting from the net energy deficit, as has been effectively applied previously in demonstrating the absence of independent benefits inherent to modified alternate-day fasting (when limited energy intake is permitted on fasting days; 19, 20). 
Given that intermittent fasting is hypothesized to exert independent benefits via fastingmediated mechanisms, it is remarkable that only one previous human trial has attempted to isolate such effects by extending the post-absorptive period sufficient to fully initiate those mechanisms (a complete 24-h cycle). That study examined insulin-sensitivity by hyperinsulinemic-euglycemic clamp rather than examining the response to ingested nutrients or the effect on components of energy balance, revealing that 24-h fasting for three non-consecutive days each week reduced fat mass, blood lipids and fasted insulin more effectively than either continuous energy restriction or fasting without energy restriction in women with overweight (21). The present study provides insight using a comprehensive experimental design that contrasts strict alternate-day fasting both with and without weight loss relative to a continuous energy restriction control, thus isolating the independent and combined effects of fasting and dietary energy restriction on measures of body composition, components of energy balance, postprandial markers of metabolic health, and subcutaneous adipose tissue gene expression (for full protocol see 22). This focus on prolonged fasting but with controlled compensatory refeeding therefore addresses fundamental questions about whether this popular form of intermittent fasting improves body composition and metabolic health independent of energy balance and weight loss. We hypothesized that intermittent fasting would result in compensatory inhibition of energy expenditure but improve metabolic health markers irrespective of reductions in adiposity. 
Science

Translational

Medicine

MIAAAS

\section{Results}

\section{Body Composition}

Alternate-day fasting was no more or less effective than a matched degree of daily energy restriction in reducing body mass. However, whereas daily energy restriction (75:75) elicited weight loss almost entirely by reducing body fat mass, weight loss via alternate-day fasting (0:150) was due in equal measure to reductions in both fat and fat-free mass (Table 1 and Figure 1A). These body composition data correspond to pre-post group differences (group $\mathrm{x}$ time interaction $p<0.0001$ ) in mean \pm SD percent body fat of $-1.8 \pm 0.9 \%$ when restricting energy daily $(75: 75 ; p<0.0001)$ but only $-0.6 \pm 1.1 \%$ or $0.1 \pm 0.9 \%$ when alternate-day fasting with (0:150) or without $(\mathbf{0 : 2 0 0})$ matched energy restriction, respectively $(p=0.10 \& p=0.69)$. However, this pattern was not apparent for visceral fat mass, which was reduced by a similar extent irrespective of whether energy restriction was applied continuously or intermittently (time $p=0.003$, group x time interaction $p=0.30 ;$ Table 1).

\section{Components of Energy Balance}

The energy ingested in the form of each dietary macronutrient is presented in Figure 1B, as reported during the baseline monitoring phase and then each intervention. These changes in energy intake from baseline were different between groups, consistent with the prescribed experimental model (group $\mathrm{x}$ time interaction $p<0.0001$ ). Furthermore, the group who were asked to fast but without reducing their net energy intake (0:200) appear to have managed to do so accurately, thus facilitating the intended analysis of fasting independent of energy restriction in relation to potential compensatory responses on the other side of the energy balance equation. 
The various components of energy expenditure (resting metabolic rate, diet-induced thermogenesis and physical activity thermogenesis) are illustrated in Figure 1C and show group differences for total energy expenditure (group $\mathrm{x}$ time interaction $p=0.004$ ) due to a decrease from baseline only when alternate-day fasting was combined with energy restriction (0:150; $p=0.002$ ), with post-hoc analysis revealing a specific difference in this response relative to the group which fasted without net energy restriction $(\mathbf{0 : 2 0 0} ; p<0.003)$. Figure $\mathbf{1 C}$ shows that this pattern for total energy expenditure $\left[\mathrm{kcal} \cdot \mathrm{d}^{-1}\right]$ is partly attributable to a slight but variable reduction in resting metabolic rate (time $p=0.18$, group $\mathrm{x}$ time interaction $p=0.19$; time effects with no differences between groups were apparent for basal/fasted rates $\left[\mathrm{g} \cdot \mathrm{min}^{-1}\right]$ of carbohydrate and lipid oxidation $p=0.02 \& p=0.01$, respectively - see data file $S 1$ ) and partly due to a consistent but predictable fall in diet-induced thermogenesis based on reported macronutrient intake (group $\mathrm{x}$ time interaction $p=0.004$ ). Accordingly, the reductions in dietinduced thermogenesis with energy restriction applied either daily $(\mathbf{7 5 : 7 5} ; p<0.0001)$ or every other day $(\mathbf{0 : 1 5 0} ; p=0.0002)$ were necessarily proportionate to the prescribed reduction in energy intake.

Figure 1D presents data for post-prandial substrate oxidation following the standardized breakfast consumed before and after the intervention; the total amount of energy expended during the $3 \mathrm{~h}$ post-prandial period was systematically reduced by a similarly small amount $(7 \pm$ 16 kilocalories over 180 minutes) in all groups (time $p=0.02$ ), with no differences between groups (group $\mathrm{x}$ time interaction $p=0.36$ ). However, whereas the overall rate of substrate oxidation was not affected by fasting alone or in combination with weight loss, the rate of lipid oxidation $\left[\mathrm{g} \cdot \mathrm{min}^{-1}\right]$ over that period did increase from pre-to-post intervention in the two fasting 
groups (0:150 \& 0:200) relative to the daily energy restriction group (75:75; group $\mathrm{x}$ time interaction $p=0.047$ ).

In terms of physical activity thermogenesis, we observed divergent behavioural responses to fasting and energy restriction (group x time interaction $p=0.04$; Figure 1E), albeit without any post-hoc differences observed between pairwise group contrasts. Specifically, restricting energy intake via alternate-day fasting (0:150) resulted in a compensatory reduction in physical activity thermogenesis, primarily due to reduced spontaneous light- and moderate-intensity movements, whereas no such reductions in activity were apparent during daily energy restriction (75:75) or alternate-day fasting without energy restriction (0:200). Figure 1F shows an exploratory analysis of how the overall $\sim 100 \mathrm{kcal} \cdot \mathrm{d}^{-1}$ reduction in physical activity energy expenditure when fasting with net energy restriction (0:150) was predominantly due to lower activity during fasting periods (especially when they occurred in the second half of the day), although there was also evidence of reduced activity during fed periods (especially when they occurred during the first part of the day).

\section{Post-Prandial Metabolism}

None of the dietary interventions differentially affected fasted concentrations or postprandial responses of plasma glucose, insulin, non-esterified fatty acids, glycerol, total cholesterol or the fractions of either high- or low-density lipoprotein cholesterol (Table 2, Figures 2 \& 3). Although fasted triacylglycerol concentrations did not respond differently between treatments, the total area under curve did exhibit pre-post group differences reflecting an increase when energy was restricted daily (75:75) relative to a decrease when energy was restricted by alternate-day fasting (0:150; group $\mathrm{x}$ time interaction $p=0.02$; Table 2). 
Group differences were apparent in the pre-post response of plasma leptin concentrations (group $\mathrm{x}$ time interaction $p=0.04$ ), with the greatest decrement in the daily energy restriction group (75:75; Table 2). In contrast to these clear effects on leptin, neither energy restriction nor fasting exerted any influence on the plasma concentrations of adiponectin under overnight fasted conditions (Table 2) or on the acute response of acylated ghrelin and peptide YY to the two sequential mixed-meal tolerance tests (Figure 3C and Figure 3D). There were no differences in fasted or postprandial plasma CTX concentrations throughout the laboratory visits pre- and postintervention (Figure 3B) nor any group differences in bone mineral density (Table 1).

Overall, of the various genes pre-selected on the basis of their involvement in biological rhythms and metabolic responses to feeding, the relative changes in mRNA from pre- to postintervention did not indicate a clear response either within or between groups (Figure 4). This was evident both in the general absence of significant group $\mathrm{x}$ time interactions and by the fact that the fold-changes observed in the raw data were similar in absolute terms between groups (see data file $\mathrm{S} 1$ ). Nonetheless, it should be noted that significant group $\mathrm{x}$ time interactions $(p \leq 0.05)$ were apparent for CEBPB (CCAAT-Enhancer Binding Protein- $\beta$ ), IRS2 (Insulin Receptor Substrate 2), PER1 (Period Circadian Regulator 1) and PPARGC1A (Peroxisome Proliferator-Activated Receptor $\gamma$ Coactivator 1- $\alpha$ ), of which post-hoc analysis indicated significant within group responses for the latter two in the daily energy restriction group ( $p \leq 0.05$; 75:75). 


\section{Discussion}

The present study indicates that alternate-day fasting is less effective for reducing body fat content than a matched degree of energy restriction applied daily without fasting in lean healthy adults. This difference in fat loss despite a standardized reduction in energy intake may be at least partly explained by the compensatory decrease in energy expenditure within the group who restricted energy intake via fasting (primarily due to inhibition of physical activity thermogenesis), although this effect did not differ significantly from the other groups who did not on average reduce their physical activity. However, other than an adaptive reduction in fasted concentrations of plasma leptin proportionate to changes in fat mass, there were no meaningful effects of fasting or energy restriction (independently or combined) on systemic markers of cardiometabolic health or gut hormone responses to sequential meals. There were also no clear fasting-specific patterns in the expression of key genes in subcutaneous adipose tissue.

It has been a matter of ongoing debate whether intermittent as opposed to continuous energy restriction either preserves lean mass during weight loss $(23,24)$ or favours greater muscle protein breakdown and net catabolism $(8,25)$. Although changes in fat-free mass were not significantly different between groups in the current study, our data certainly do not support any relative preservation of lean mass when fasting but rather are more consistent with the possibility that complete fasting for a prolonged period every other day $(\mathbf{0 : 1 5 0})$ is not conducive to the retention of lean mass under conditions of net energy deficit. That interpretation would be in agreement with recent trials that have reported reductions in fat-free mass even with modified alternate-day fasting that does not require such extended periods without food $(25 \%$ of energy requirements allowed on fasting days), albeit also no differently than daily energy restriction in these studies $(20,26)$. In addition, one recent study has even reported that diet-induced 
reductions in fat-free mass can be offset by only slightly increasing the daily fasting period from 18-20 $\mathrm{h}$ (27). It therefore appears that losses of fat-free mass are possible even with less protracted periods of limited nutrient delivery than implemented in the present study and so further research is needed to examine the potential for a more sustained catabolic state to result in atrophy of lean soft tissue mass or indeed to improve anabolic sensitivity (16).

Although lean soft tissue mass includes components such as water, glycogen and the nonlipid component of adipose tissue (the latter being automatically reduced by fat loss; 28), prescan fluid and food intake were carefully standardized in this experiment and any remaining effect on the lean portion of adipose tissue would be relatively minor. Indeed, a recent trial reported that late time-restricted feeding (fasting 2000-1200 h daily) reduced appendicular fatfree mass in particular, which probably reflects losses specific to skeletal muscle (29). It therefore remains a possibility that at least some of the individuals who were restricting energy intake via alternate-day fasting in the present study were experiencing a reduction in skeletal muscle mass, which might erroneously be interpreted as successful dieting if using common weighing scales. Losing metabolically active fat-free mass (especially skeletal muscle) is generally an undesirable outcome for physical function, cardiometabolic health, and for sustaining an energy deficit with minimal adaptation in metabolic requirement. Instead, intermittent fasting is generally adopted by individuals hoping to achieve weight loss in terms of reducing body fat content and yet the present study clearly illustrates that continuous daily energy restriction is more effective in achieving that outcome. It is notable, however, that visceral fat mass responded similarly to intermittent versus continuous energy restriction, as that particular compartment of adipose tissue is ordinarily highly correlated both with 
cardiometabolic risk and with the sub-cutaneous fat depot that did respond so differently to the interventions in this study (30).

In terms of metabolic rate, it is noteworthy that there was not a larger or more consistent difference between groups either under basal/fasted conditions or in response to breakfast especially given that fasting and energy restriction differentially affected changes in both body mass and composition, which automatically dictate metabolic requirements. Notably, this finding persists irrespective of whether the rates of metabolism and substrate oxidation are expressed in absolute terms $\left(\mathrm{kcal} \cdot \mathrm{d}^{-1}\right.$ and $\left.\mathrm{g} \cdot \mathrm{min}^{-1}\right)$ or relative to either body mass or fat-free-mass; any effect of fasting or energy restriction in terms of adaptive thermogenesis is therefore likely to be small and not markedly different between groups.

Beyond the above relatively trivial responses of resting metabolic rate and diet-induced thermogenesis, a major focus of this experiment and an important potential contributor to the decrement in total daily energy expenditure is the compensatory behavioural response. Indeed, the energy expended via physical activity is the most variable and malleable component of energy expenditure and thus has the greatest potential to undermine an imposed energy restriction. It is therefore surprising that no past research had objectively quantified the response of this variable specific to alternate-day fasting. Several past studies have monitored aspects of physical activity during other forms of intermittent fasting but even these few studies were designed to focus on isolating metabolic responses and so tended to advise participants not to consciously alter their physical activity during the interventions $(20,21,31,32)$. However, structured exercise is only one aspect of physical activity and sub-conscious behavioural adaptations in non-exercise activity thermogenesis (NEAT) are an important compensatory response to fasting. 
In the present study we objectively quantified all aspects of exercise and non-exercise physical activity thermogenesis under free-living conditions in which participants were given to understand that, far from consciously avoiding unplanned behaviour changes, they should behave naturally. Our observation of reduced physical activity when restricting energy intake via alternate-day fasting but without any such reductions when energy restriction or fasting were applied separately is inconsistent with a recent trial that found activity energy expenditure to be very stable during 4 weeks of alternate-day fasting relative to a non-intervention control group (thus not matched for energy restriction; 18) or after two weeks of early time-restricted feeding (16). However, behavioural compensation has been noted in past trials involving weight loss or daily caloric restriction but without fasting (33). In terms of isolating the effect of fasting per se on physical activity, two previous investigations into daily breakfast omission in lean and obese adults corroborate the current findings given that light- and moderate-intensity physical activity were also lower with extended fasting in those studies, particularly during the morning period when participants were post-absorptive $(34,35)$. Here we used the same tool to objectively measure physical activity thermogenesis and so it is similarly possible to explore the temporal pattern of behavioural compensation specific to fasted and fed periods.

To consider what might explain the apparent differences in physical activity, it is important to note that the responses to the intervention cannot be explained simply by reactive changes in the times participants rose in the morning or went to bed at night depending on whether they were fasting, because these times remained within six minutes of the times recorded at baseline. Equally, the energy expended directly preparing (or not preparing) food is unlikely to account for overall changes in physical activity as the overall energetic contribution of cooking activities is minimal (36). It is also conceivable that systematic bradycardia or 
tachycardia may respectively explain the lower energy expenditure with fasting or the higher energy expenditure when refeeding (particularly considering the increase in physical activity specific to fed periods in the group who avoided energy restriction). This is also unlikely, however, because extreme (even maximal) overeating only increases resting heart rate by $~ 6$ beats $\cdot \mathrm{min}^{-1}$ in the immediate post-prandial period (37) and by $\sim 2$ beats $\cdot \mathrm{min}^{-1}$ after a week of energy surplus (38). Recent work has also now reported anticipatory behavioural modification even outside the period of dietary intervention, which cannot therefore be attributed to changes in heart rate during the fast (39). Moreover, the changes in physical activity reported here were all primarily due to light- and moderate-intensity movements, for which the branched model equations used to calculate energy expenditure are weighted heavily towards accelerometry unless heart rate is increased markedly above rest.

It therefore seems reasonable to conclude that behavioural compensation in low-tomoderate intensity physical activity is indeed responsible for the divergent responses of physical activity thermogenesis between groups in this study, consistent with the hypothesis that subconscious engagement in spontaneous movements is reduced by the interaction of alternate-day fasting with weight loss but not by fasting per se. This may therefore be a direct influence of low nutrient availability due to fasting, although our results indicate that not all the reduction in physical activity occurred during fasting periods (with some reduction in energy expenditure during fed periods, especially when they occurred during the first part of the day). The latter may indicate an anticipatory reduction in physical activity behaviours in advance of the impending dietary restriction (39). Future research is therefore warranted to examine both whether there is any carry-over effect from the overfeeding period that preceded both afternoon fasting and 
morning feeding and also whether early- or late-time restricted feeding may differ in their potential to elicit this behavioural response.

Understanding the effects of intermittent fasting on post-prandial metabolic control has recently been identified as a research priority $(1,9)$. Post-prandial measures provide a better indication of disease risk than basal/fasted blood samples $(40,41)$, and simultaneously provide a more valid reflection of the fed state that generally predominates for the majority of each 24-h cycle $(2,3)$. Notably, the few studies to have begun examining meal responses to intermittent fasting diets have identified clear benefits in terms of reducing post-prandial glycemia (42), insulinemia (15) and triglyceridemia (43), albeit without any improvement in whole-body insulin sensitivity whether assessed via hyperinsulinemic-euglycemic clamp (21) or oral glucose tolerance test (44). Most recently, however, early time-restricted feeding was shown to improve post-prandial skeletal muscle glucose and branched-chain amino acid uptake in healthy men independent of weight loss (16). In view of these recent findings, it is notable that fasted and post-prandial metabolites were generally unresponsive to energy restriction in the present study, particularly in light of the post-prandial substrate oxidation data, which indicated that the fasting interventions increased post-prandial lipid oxidation.

One possible explanation for the absence of response in relation to systemic indices of cardiometabolic health in this study is that an intervention longer than three weeks may be required to elicit meaningful changes in these outcomes, although it has been argued that such responses are usually evident within 2-4 weeks (4). That latter interpretation would certainly be consistent with recent studies of intermittent fasting that show effects on insulin sensitivity and glycemic control within $1-5$ weeks $(15,42)$. A more likely reason why markers of cardiometabolic health were not improved by fasting or weight loss in the present study may be 
that participants were not overweight at baseline. Specifically, positive effects of energy restriction on metabolic profile may be secondary to clearance of ectopic lipids (45), which may not yet have accumulated substantially in the ostensibly healthy individuals tested here. Further research should therefore repeat this experiment in an overweight or obese population.

Leptin is secreted from adipose tissue in proportion to fat mass (46). It is therefore understandable that the varied decrements in fat mass observed in the two energy restriction trials in this study (75:75 \& $\mathbf{0 : 1 5 0})$ were mirrored by group differences in the pre-post response of fasted concentration of plasma leptin. This pattern is consistent with the physiological role of leptin as part of a negative feedback loop to sustain endogenous energy reserves in the face of sustained negative energy balance (47), thus validating the established relationship between leptin and fat mass irrespective of whether weight loss is elicited by continuous energy restriction or intermittent fasting. It might therefore be speculated that the greater fat loss in the daily energy restriction group (75:75) might lower satiety more so than the alternate-day fasting groups in order to minimise further reductions in fat mass or even restore the lost energy reserves. When considering this effect on leptin alongside the absence of group differences in acylated ghrelin and peptide YY, it appears that that energy restriction can elicit an adaptive neuroendocrine response via the basal concentration of tonic (longer-term) satiety hormones such as leptin but without compensation in the acute concentrations of episodic appetite hormones to daily meals, although leptin may act synergistically to potentiate the response of acute hormones $(48,49)$.

Although not pre-specified as an outcome measure in our clinical trial registration (www.clinicaltrials.gov NCT02498002), an opportunity arose to measure plasma concentrations of carboxy-terminal telopeptide of type I collagen crosslinks (CTX) as a marker of bone 
resorption, thus allowing an exploratory analysis of bone turnover with intermittent fasting relative to the bone mineral density data obtained from the DXA scans in this study. Our observation that neither plasma CTX or bone mineral density differed between treatments may simply reflect that it requires longer than 6 months to reveal measurable changes in bone turnover via DXA, yet even 6 months of alternate-day fasting does not appear to alter bone mineral density or fasted plasma CTX concentrations (50). Nonetheless, CTX rapidly responds to under 4 weeks of intensive exercise with restriction and subsequent replacement of carbohydrate alone (even without net energy restriction; 51). It may therefore be that the additional stimulus of exercise and restriction of carbohydrate in particular may be required to alter bone metabolism during interventions lasting only a few weeks.

To the authors' knowledge, this trial provides a report of gene expression in human adipose tissue within the context of alternate-day fasting. Rather than interpreting the ostensible response of a few genes in isolation, illustrating such data using a heat map renders it possible to consider the overall pattern of responses across all the genes that operate in concert. From that perspective, it may be worth noting that fasting with net energy restriction (0:150) indicates consistent downregulation of almost all genes analyzed, with the few exceptions that were markedly upregulated all being the same genes that are markedly downregulated after fasting without net energy restriction (0:200) and also all implicated in inflammation (CEBPB, NAMPT/visfatin \& Tumor Necrosis Factor- $\alpha$ ). These few observations notwithstanding, the overall lack of effects on gene expression in the present study of lean individuals may indicate that fasting and energy restriction for several weeks do not exert any substantial influence at the genetic level in human subcutaneous adipose tissue. This absence of effect may therefore be tissue-specific because skeletal muscle does exhibit altered expression of certain metabolic genes 
(but not core clock genes) in response to time-restricted feeding (52). It may be that any effects of fasting on adipose tissue gene expression are short-lived and so do not persist following even one day of habitual energy intake, which in the present study was standardized within each participant $24 \mathrm{~h}$ prior to follow-up samples in order to replicate conditions preceding the corresponding baseline samples. Alternatively, a more prolonged or intensive intervention that elicits more profound reductions in fat mass may be necessary before effects on adipose tissue gene expression can be detected.

There are several limitations to our study that arise primarily due to the practical constraints inherent to intensive human intervention trials. Although groups were well-matched for physiological characteristics at baseline, the proportion of males and females was not equal between groups (table S1) and the sample size was slightly reduced for adipose tissue gene expression as adipose biopsies were an optional procedure for which few volunteered (although the small/similar absolute fold-changes in gene expression indicate that no physiologically meaningful differences have gone undetected simply due to statistical power). Similarly, no participants elected to provide skeletal muscle biopsies; measures of muscle protein synthesis would have been informative in view of the fat-free mass data reported here. In relation to the free-living intervention, it should be acknowledged firstly that compliance was self-reported (so would have benefitted from objective verification via continuous glucose monitoring; 34,35 ) and secondly that additional effects may have become evident had it been feasible to sustain this intervention beyond 3 weeks. The post-prandial measures of triacylglycerol and glycerol should also be interpreted with caution because a high degree of variability was apparent in measured values, yet the effects reported here in relation to triacylglycerol total area under curve are consistent with a previous study in which fasting on 2 consecutive days per week for $\sim 8$ weeks 
reduced post-prandial triglyceridemia (43). Last, not all metabolic responses to the second meal had returned to basal levels within the 2-h post-prandial measurement period. It would therefore be interesting to have extended the monitoring period later into the evening and, ideally, to have documented a complete 24-h diurnal cycle.

The fact that intermittent fasting exerted no greater effect than continuous daily energy restriction on anthropometric, metabolic, and behavioural outcomes in this study may in part be a consequence of the alternating daily nature of the fasting periods. Specifically, the transition between fasted and fed states at $1500 \mathrm{~h}$ on alternate days may not be conducive to the entrainment of diurnal rhythms within the human circadian timing system; the signalling effects of high or low nutrient availability may then be compromised because metabolic and behavioural food anticipatory activity cannot be aligned to a regular meal pattern in each daylight period (53). It may therefore be that intermittent fasting models such as alternate-day fasting or the " $5: 2$ " diet that do not repeat every 24 hours necessarily have less potential to optimise physiological function than models in which fasting is scheduled at the same time each day (such as time-restricted feeding). However, given that human physiology operates to defend against the desired energy deficit that is the objective of dieting, there may still be some value in deliberately misaligning and thus impairing that natural protective response via a more erratic fasting schedule - perhaps in overweight or obese individuals for whom there is more excess body fat to lose or pre-existing health conditions to rectify.

Although the intermittent fasting regimen employed here was inferior for reducing body fat relative to daily energy restriction in terms of our completers-only analysis, participants allocated to the 0:150 group nonetheless appeared to find adoption and adherence reasonably acceptable (or at least tolerable, with only one withdrawal) and did experience significant albeit 
more modest reductions in adiposity. Alternate-day fasting may still therefore represent a viable even if sub-optimal weight loss option for those who find this pattern relatively easy to adopt and adhere to within their wider lifestyle, perhaps in combination with countermeasures targeting lean tissue mass (such as resistance exercise). Even accepting that alternate-day fasting may not be sufficiently feasible in the specific form described here or may not be justified by the overall risk-benefit analysis, an improved basic science understanding of intermittent fasting can inform related approaches (development of novel ingredients, periodization of particular nutrients or exercise, fasting mimicking diets, and so on) with a view to maximizing potential advantages whilst minimizing any disadvantages.

In summary, for lean healthy adults, continuous daily energy restriction (traditional diet advice) results in meaningful weight loss that is almost entirely attributable to reduced adipose tissue mass. By contrast, using alternate-day fasting to elicit the same energy deficit can spontaneously inhibit physical activity relative to the usual pre-diet engagement in such behaviour and so less effectively reduces fat mass than continuous daily energy restriction. These are generally undesirable responses linked to poor long-term health outcomes, although no shortterm changes in metabolic health were apparent in the lean population reported here. The main practical message for individuals to consider if planning to use alternate-day fasting for the purpose of weight loss or health gain is thus to consciously insert opportunities for physical activity alongside the intermittent fasting regimen in order to maintain energy expenditure and maximize potential beneficial effects on body composition. 


\section{Materials and Methods}

\section{Study Design}

We conducted a randomized controlled trial in which 36 lean healthy adults were randomly allocated into three experimental conditions following a 4-week baseline monitoring phase (habitual diet and physical activity). One group $(n=12)$ served as a continuous dietary energy restriction control by being prescribed $75 \%$ of their habitual energy intake each day $(25 \%$ daily energy restriction; 75:75); a second group $(n=12)$ were prescribed a matched degree of dietary energy restriction but achieved via alternating 24-h periods of complete fasting and consuming $150 \%$ of their habitual energy intake (25\% net energy restriction; 0:150); a third group $(n=12)$ were prescribed a matched pattern of complete fasting every other 24 hours but consuming $200 \%$ of their habitual energy intake on fed days (no net dietary energy restriction; 0:200).

Critically, the two fasting groups (0:150 \& 0:200) consumed no energy-providing nutrients whatsoever during fasting periods and transitioned from fasted to fed state and vice versa at $1500 \mathrm{~h}$ on a daily basis. This time was selected for a number of reasons: it provides a consistent fasting duration of precisely $24 \mathrm{~h}$, thus sufficient to elicit the desired fasting-mediated mechanisms whilst also standardized between fasted and fed periods (rather than a more variable fasting duration if dictated by sleep times); our recent work revealed diurnal rhythms in human skeletal muscle transcriptomics and lipidomics that exhibit peak accumulation of relevant gene transcript and lipid metabolites at $1600 \mathrm{~h}(54,55)$, so the metabolic switch between the fed and fasted state would be complete by $1600 \mathrm{~h}$ each day (11); effects can be attributed to fasting as opposed to any systematic shift in circadian rhythms because the time-restricted feeding alternates between the first and second part of each day-night cycle; the transition occurs at 
approximately the mid-point of each waking period, allowing equally weighted contrasts between the metabolic and behavioural responses in each half of the day; and our pilot testing suggests that participants find it relatively feasible to fast for this duration given that there is no complete waking period without food.

In terms of outcome measures, recent reviews on this topic have highlighted the need for trials investigating the components of energy balance under free-living conditions in order to understand the distinct compensatory metabolic and behavioural responses to fasting per se as opposed to mere energy restriction $(1,9)$. In particular, there is an outstanding need to establish the effects of regularly fasting for an extended period on the preservation of fat-free mass (and thus resting metabolic rate during weight loss); compensatory behavioural adjustments in physical activity thermogenesis; and alterations in metabolic control both under fasted conditions and in the fed state. Below we therefore present data for three primary outcomes, namely changes in body composition, components of energy balance, and post-absorptive/post-prandial measures of systemic metabolites and regulatory responses to sequential mixed-meal tolerance tests (breakfast and lunch) applied pre- and post-intervention.

\section{Experimental Model \& Subject Details}

Sampling \& Recruitment - This study involved the recruitment of human volunteers and thus obtained a favourable opinion from a National Health Service Research Ethics Committee (reference: 15/SW/0007). Participants were recruited via local advertisement in the South West of the United Kingdom between 2015 and 2018 (trial first advertised May $20^{\text {th }} 2015$ and first participant enrolled June $17^{\text {th }} 2015$ ) in order to obtain a total sample of 36 lean and purportedly healthy men and women. This sample size is consistent with previous studies in this area that 
have provided reliable and useful data, so was justified using a priori statistical power estimates based on studies of similar duration to have examined the outcome measures in question. For example, one study (56) observed a decline in resting metabolic rate following 21 days of daily energy restriction $\left(1898 \pm 262\right.$ versus $\left.1670 \pm 203 \mathrm{kcal} \cdot \mathrm{day}^{-1}\right)$, which would therefore have an $80 \%$ probability of detection at $p \leq 0.05$ with $n=33$. In relation to other outcomes in this study, a previous study (57) reported a reduction in 2-hour post-prandial plasma glucose concentrations following 10 days of daily energy restriction $\left(10.7 \pm 3.6\right.$ versus $\left.7.1 \pm 3.0 \mathrm{mmol} \cdot \mathrm{l}^{-1}\right)$, in which case $n=27$ would be required to achieve beta 0.8 and alpha 0.05 . Collectively, it was therefore deemed that $\sim 30$ participants would be adequate to detect meaningful treatment effects in the present study and recruitment proceeded on a rolling basis until at least 12 had completed each experimental condition given that our primary hypotheses concern the physiological responses of actually fasting, so a completers-only analysis is appropriate.

To be eligible upon volunteering, participants had to first be classified as lean based on body mass index (BMI; 20.5-24.9 $\mathrm{kg} \cdot \mathrm{m}^{-2}$ ), which was subsequently confirmed upon their first laboratory visit using sex-specific fat mass index (FMI) obtained from a dual-energy x-ray absorptiometry (DXA) scan. Values of $\leq 7.5 \mathrm{~kg} \cdot \mathrm{m}^{-2}$ and $\leq 11.0 \mathrm{~kg} \cdot \mathrm{m}^{-2}$ were classified as lean for males and females, respectively. Further inclusion criteria for this study were that participants must be: aged 18-65 years; weight stable $( \pm 3 \mathrm{~kg}$ ) for prior 6 months; able and willing to comply with study procedures; willing to undertake required fasting durations; and have the capacity to provide informed consent. Exclusion criteria were as follows: body mass >120 kg; engagement in fasting practices within 3 months of start date; recent or planned change in diet/physical activity habits; evidence of disordered eating as assessed using the EDE-Q 6.0 (Fairburn, 2008); diagnosis with diabetes or other metabolic health disturbances; ongoing medical condition or 
treatment which may interfere with study variables; menopausal; pregnant, recently pregnant or planning to become pregnant (within 3 months) or currently breastfeeding; having donated blood within the last 3 months; lack of capacity/language skills to independently follow the protocol; unable to consume test meals due to intolerances/dietary preferences (vegan, gluten, milk proteins); or any other behaviour or condition that introduces bias to the experiment or poses undue personal risk. Baseline characteristics of the 36 individuals who completed the study are reported in table S1. A total of 97 individuals were formally screened for eligibility, of whom 42 met the inclusion criteria and were consented to complete the first laboratory visit. During the 4week baseline monitoring control phase, three individuals withdrew from the trial (citing illness, work, or relocation) and two more were excluded from the trial for data outside the inclusion criteria (not weight-stable at baseline and implausibly low energy intake). Post-randomization only one participant withdrew from the study (0:150 group), citing difficulty fasting. Hence the final sample size of completers was $n=36$ (see supplementary participant flow diagram; fig. S1).

Experimental Design - A randomized controlled trial (www.clinicaltrials.gov NCT02498002; trial registered July $15^{\text {th }} 2015$ ) with an independent measures, parallel groups design was adopted to contrast the effects of intermittent fasting with and without weight-loss relative to standard daily energy restriction. Specifically, all volunteers completed a 4-week baseline monitoring phase during which they consumed and recorded their habitual diet without consciously modifying any aspect of their lifestyle. One week later participants were randomly assigned to a 4-week dietary intervention using a blocked randomisation scheme with a 1:1:1 allocation and stratified according to fat-mass index and physical activity level (PAL), thus ensuring a relatively even distribution of more (PAL: $\geq 1.75)$ and less (PAL: $<1.75)$ active participants in each group. 
This randomization scheme, including block sizes and sequences, was generated by an individual who was not involved in participant recruitment or aware of participant identification codes, with details of the allocation sequence concealed from all who were involved with participant recruitment and code assignment in order to minimise any risk of bias. Participants completed a pre-intervention laboratory visit, after which they received their group assignment and adhered to the prescribed dietary intervention for 20 days (with monitoring of free-living diet and physical activity during), before a post-intervention laboratory visit to follow-up pre-intervention measures. The three experimental conditions were as follows: Daily Energy Restriction (75\% of habitual diet every 24 hours; 75:75); Alternate-Day Fasting with net Energy Restriction (alternating 0\% and $150 \%$ of habitual diet every other 24 hours; 0:150); Alternate-Day Fasting without net Energy Restriction (alternating 0\% and 200\% of habitual diet every other 24 hours; 0:200).

Protocol - Following the provision of written informed consent, eligibility was initially assessed using a series of self-report questionnaires together with a BMI calculation. Eligible participants then undertook the 8 -week protocol shown in fig. S2. For all laboratory sessions, participants abstained from caffeine, alcohol, smoking and strenuous exercise throughout the preceding 24 hours, and also standardized their dietary intake on a within-participant basis. Following an overnight fast (minimum 10 hours), participants reported to the laboratory at $0730 \mathrm{~h}( \pm 1 \mathrm{~h})$ having consumed $500 \mathrm{ml}$ of water upon waking. For female participants, laboratory sessions were scheduled to coincide with the follicular phase of their menstrual cycle (3-10 days after onset of menses). 
Baseline Laboratory Visit 1 - This visit provided a reference point for examining the stability of body mass, as an indicator of overall energy balance, throughout the ensuing 4-week control/monitoring phase in which habitual dietary intake and physical activity were quantified. In addition, this visit served to familiarise participants with key procedures to improve reliability over subsequent laboratory sessions. A urine sample was collected when voiding prior to measurements of height and body mass to ensure adequate hydration for these measurements (urine specific gravity $<1.020$ and osmolality $<900 \mathrm{mOsm}$ ). Following a 20 -minute rest in a semi-recumbent position, resting metabolic rate and substrate oxidation were then measured via indirect calorimetry of expired gas samples, after which a fasted blood sample was drawn. To conclude this session, a submaximal treadmill protocol was undertaken to individually calibrate the physical activity monitors being used throughout the study (Actiheart, Cambridge Neurotechnology). Before departing, participants were given the materials to capture free-living measurements of dietary intake and physical activity.

Control/Monitoring Phase - During this phase, both energy intake and physical activity energy expenditure were measured concurrently in four designated monitoring windows of three consecutive days each. Each of these windows was separated by at least two days from any other and the final window covered the three days leading up to the second laboratory visit to ensure compliance with standardisation procedures. Physical activity energy expenditure and intensity were measured using individually-calibrated Actiheart monitors and energy intake using a weighed record of food and fluid intake. Energy balance was verified by maintaining a stable body weight $(\leq 1.0 \mathrm{~kg}$ increase or decrease) between the first and second laboratory visits.

Pre-intervention Laboratory Visit 2 - Participants returned to the laboratory following the control/monitoring phase for measurement of a series of fasted and postprandial outcomes. 
Adequate hydration status was again ascertained via urinary biomarkers upon arrival prior to measuring body mass. Fasting measurements of resting metabolic rate and substrate oxidation were then obtained before an intravenous cannula was fitted to an antecubital vein. At this stage, an initial venous blood sample was drawn to provide fasted concentrations of relevant metabolites and hormones. A small sample of adipose tissue was obtained from volunteers who opted-in to that procedure (in which case a repeated venous blood sample was taken afterwards to serve as the baseline for the meal response tests). Participants then completed the first of two sequential mixed-macronutrient meal tests: a homogenous porridge meal (meal 1; breakfast) and a meal-replacement shake (meal 2; lunch). The morning post-breakfast period involved the collection of expired gases for indirect calorimetry along with venous blood samples, whereas the afternoon post-lunch period only involved blood sampling.

Intervention Phase - Following pre-intervention laboratory visit 2, a 6-day wash-out period occurred prior to commencing the interventions - this was done both to avoid prolonged periods of lifestyle monitoring and to maintain an interval of 4 weeks between pre- and post-intervention tests. During fasting cycles participants were only permitted water, herbal teas and black tea/coffee with no sugar (unsweetened energy-free drinks). During feeding cycles, and throughout the daily energy restriction intervention, participants were prescribed the same meals as they reported habitually consuming during their baseline monitoring phase but with quantities proportionately modified to provide $75 \%, 150 \%$ or $200 \%$ of their habitual energy intake. Energy intake and physical activity were also monitored over the first and last 6 days of the intervention period - the former to quantify compliance and the latter to examine behavioural compensation in energy expenditure. 
Post-intervention Laboratory Visit 3 - After the completion of 20 consecutive 24-hour dietary cycles plus one wash-out day of replicating the standardised diet and activity from before the pre-intervention visit, participants returned to the laboratory and repeated the protocol outlined earlier for laboratory visit 2 .

\section{Outcome Measures}

Body Composition - Post-void body mass was measured to the nearest $0.1 \mathrm{~kg}$ using a sliding balance scale (Weylux 424) and height was measured to the nearest $0.1 \mathrm{~cm}$ using a wall-mounted stadiometer (Seca Stadiometer). Body composition was assessed using a DXA scan (QDR Discovery W, Hologic) conducted in accordance with the manufacturer's instructions. Prior to each exposure a quality control procedure was executed in which a spine phantom with known radiographic attenuation properties was scanned to ensure adequate performance. This was accompanied by a background radiographic uniformity test at regular intervals, in which a whole-body scan was completed whilst the scanning table was empty to ensure proper functioning and monitor changes in background radiation levels. All DXA scans were obtained at the end of data collection sessions to provide greater control over hydration status and tissue glycogen content. Before scans, participants voided, wore the same lightweight clothing and removed shoes along with any heavy items and jewellery.

Dietary Intake - Participants were provided with a set of compact kitchen scales (Pocket Pro 2000, Smart Weigh) and a logbook with which all food and drink items were recorded. A member of the research team discussed best practice with participants and emphasised the level of detail required. Weighed records were analysed (Nutritics version 5.031) to determine energy and macronutrient intake. Free-living diet-induced 
thermogenesis was then estimated based on these self-reported food diaries based on the established constants for the thermogenic effect of each macronutrient ingested (58).

Physical Activity - Physical activity was measured using Actiheart monitors (Cambridge Neurotechnology). These monitors were individually calibrated using a treadmill protocol involving four 3-minute stages of incremental treadmill locomotion with concurrent measurements of heart rate and energy expenditure (indirect calorimetry of expired gas samples) to yield a heart rate-physical activity intensity regression equation upon which estimates were based. The times at which participants rose in the morning and went to bed at night were determined based on visual inspection of individual daily physical activity traces to identify when physical movements commenced and ceased at the beginning and end of each waking phase (this analysis was completed by an individual blinded to treatment allocation).

Meal Tests - Two successive meal tests were completed pre- and post-intervention. Both meals were prescribed to provide one-third of resting metabolic rate, as measured preintervention. Meal 1 was a homogenous porridge meal $\left(1.31 \mathrm{kcal} \cdot \mathrm{g}^{-1} ; 59 \%\right.$ carbohydrate, $29 \%$ fat, $12 \%$ protein) composed of golden syrup flavour instant oats (Sainsbury's), whole milk (Tesco) and white granulated sugar (Silver Spoon). This was cooked in a microwave and cooled for 10 minutes before being consumed in its entirety within a 10minute eating opportunity following a pre-meal blood draw. Meal 2 took the form of a liquid meal-replacement supplement $\left(1.50 \mathrm{kcal} \cdot \mathrm{ml}^{-1} ; 54 \%\right.$ carbohydrate, $30 \%$ fat, $16 \%$ protein) (Ensure Plus; Abbott Nutrition). This was consumed following a pre-meal arterialised-venous blood draw within a 5-minute feeding window commencing 3.5 hours after the consumption of meal 1 . 
Indirect Calorimetry - Resting metabolic rate and substrate oxidation were measured using indirect calorimetry of expired gas samples (59). In each instance, three consecutive 5-minute samples were taken in accordance with best practice guidelines $(60)$, with the values from two or more samples that agree to within $100 \mathrm{kcal} \cdot \mathrm{day}^{-1}$ taken as an arithmetic average.

Blood Sampling and Analysis - At the pre- and post-intervention visits all blood samples were procured by means of an intravenous cannula. To permit the sampling of arterialised-venous blood, for 10 minutes prior to arterialised-venous sampling intervals participants were asked to place the hand of their cannulated arm into a heated-air box (University of Vermont), the internal environment of which was held steady at $55^{\circ} \mathrm{C}$. Samples were drawn and dispensed into an EDTA-coated tube for processing before the cannula was flushed with $0.9 \%$ saline to keep it patent. Analysis of plasma samples for concentrations of metabolites was performed using an automated analyser (RX Daytona; Randox Laboratories) and commercially available reagents (Randox Laboratories). Plasma insulin and leptin concentrations were determined using commercially available ELISAs (Mercodia). For the analysis of acylated ghrelin, $1 \mathrm{~mL}$ of EDTA-treated whole blood was treated with $50 \mu \mathrm{L}$ of a p-hydroxymercuribenzoic acid solution (prepared as $100 \mathrm{mM}$ concentrate solution in potassium phosphate buffer containing $1.2 \% 10 \mathrm{~m}$ $\mathrm{NaOH}$ ). Samples treated in this way were analysed for acylated ghrelin using commercially available ELISAs (Merck-Millipore). Total PYY was analysed in plasma samples using commercially available ELISAs (Merck-Millipore). Plasma C-terminal telopeptide of type I collagen (CTX) concentrations were analysed using commercially available ELISAs (Immunodiagnostic Systems). For all assays, where concentrations fell 
below the limit of detection for the assay, values were supplanted by the limit of detection, as specified by the assay manufacturer.

Adipose Tissue Sampling and Analysis - Subcutaneous adipose tissue biopsies were performed under local anaesthesia (1\% lidocaine (lignocaine)) from the area around the waist approximately $5 \mathrm{~cm}$ lateral to the umbilicus using a $14-\mathrm{G}$ needle using an aspiration technique with follow-up biopsies sampled from the opposite side. The sample was cleaned with isotonic saline and any clot was manually removed. After weighing the sample, it was homogenized in $5 \mathrm{~mL}$ of Trizol (Invitrogen) and placed on dry ice before being stored at $-80^{\circ} \mathrm{C}$. Subsequently, samples were defrosted and vortexed, before 0.1 $\mathrm{mL}$ of 1-Bromo-3-Chloropropane per $1 \mathrm{~mL}$ of Trizol was added. After shaking the mixture vigorously for $30 \mathrm{~s}$, samples were incubated at room temperature for $3 \mathrm{~min}$ and then centrifuged at $10,000 \mathrm{~g}$ for $15 \mathrm{~min}$ at $4{ }^{\circ} \mathrm{C}$. The aqueous phase was removed and mixed with $0.5 \mathrm{~mL}$ ice-cold isopropanol per $1 \mathrm{~mL}$ of Trizol and stored overnight at $-20^{\circ} \mathrm{C}$ to precipitate RNA. Samples were centrifuged at $10,000 \mathrm{~g}$ for $10 \mathrm{~min}$ at $4{ }^{\circ} \mathrm{C}$ and the supernatant was discarded. The remaining pellet was washed in $1 \mathrm{~mL}$ of $75 \%$ ethanol per $1 \mathrm{~mL}$ of Trizol and centrifuged at $10,000 \mathrm{~g}$ for $10 \mathrm{~min}$. Supernatant was removed once more and the pellet was air-dried for 10 min before being suspended in $30 \mu \mathrm{L}$ of RNasefree water. Each sample was quantified by spectrophotometry using a NanoDrop One (Thermo Scientific), with $5 \mathrm{ng}$ of total RNA reverse transcribed using a high-capacity cDNA reverse transcription kit (SuperScript III, Invitrogen). A $100 \mu \mathrm{L}$ reaction mix comprising 200 ng of cDNA, $50 \mu \mathrm{L}$ of Universal Mastermix (Applied Biosystems) and RNase-free water, was used for each sample. The following assays, obtained from Applied Biosystems, were run on a real-time PCR system (7900HT, Applied Biosystems) 
according to manufacturer's guidance: CLOCK (Hs00231857_m1), CRY1 (Hs00172734_m1), NPAS2 (Hs00231212_m1), PER1 (Hs00242988_m1), CREB1 (Hs00231713_m1), APLN (Hs00175572_m1), C/EBP alpha (Hs00269972_s1), C/EBP beta (Hs00942496_s1), LEPTIN (Hs00174877_m1), AKT2 (Hs00609846_m1), IRS1 (Hs00178563_m1), IRS2 (Hs00275843_s1), PIK3R1 (Hs00381459_m1), IGF1R (Hs00609566_m1), SIRT1 (Hs01009006_m1), Slc2a4/GLUT-4 (Hs00168966_m1), FASN (Hs00188012_m1), MLXIPL/ChREBP (Hs00263027_m1), NAMPT/visfatin (Hs00237184_m1), SREBF1/SREBP1C (Hs01088691_m1), PDK4 (Hs01037712_m1), ACACA (Hs01046047_m1), ACADM (Hs00936580_m1), ANGPTL4 (Hs01101127_m1), CIDEC/FSP27 (Hs01032998_m1), CPT1 alpha (Hs00912671_m1), FABP4 (Hs01086177_m1), HADHB (Hs01027270_g1), LIPE/HSL (Hs00193510_m10), LPL (Hs01012571_m1), PLIN2 (Hs00605340_m1), PNPLA2/ATGL (Hs00386101_m1), PNPLA3 (Hs00228747_m1), PPARG (Hs01115513_m1), CIDE-A (Hs00154455_m1), IL-6 (Hs00985639_m1), PARP1 (Hs00242302_m1), TNF-a (Hs99999043_m1), ADIPOQ/adiponectin (Hs00605917_m1), PRKAA1 (Hs01562315_m1), PRKAA2 (Hs00178903_m1), PPARGC1A (Hs00173304_m1), SIRT3 (Hs00953477_m1), UCP2 (Hs01075227_m1). Relative quantification of the genes of interest was performed using the comparative CT method as previously described (61). The expression of all these genes were first normalized for the geometric mean of PPIA/Cyclophilin A (Hs04194521_s1) and PGK1 (Hs99999906_m1) which were used as endogenous controls for calculation of $\Delta \mathrm{Ct}$. The comparative threshold cycle $(\mathrm{Ct})$ method was then used to process the data where $\Delta \mathrm{Ct}=\mathrm{Ct}$ target gene $-\mathrm{Ct}$ endogenous control, with data subsequently normalized to an internal calibrator (a nominated baseline sample broadly 
displaying higher $\mathrm{Ct}$ values across all genes) for the relevant quantification using $\Delta \Delta \mathrm{Ct}$, and then to each participant's own baseline expression of each gene for calculation of the relative changes presented in Figure 4.

Urine Collection and Analysis - Urine samples collected at the outset of each laboratory session were analysed for specific gravity via refractometry (SUR-NE Clinical) and osmolality via the freezing-point depression method (Micro Osmometer 3300; Advanced Instruments). Throughout the 3-hour postprandial period following meal 1, total urine output was also collected in order to correct rates of energy and substrate metabolism for protein oxidation. Urinary urea concentration was determined using an automated analyser, as described above for plasma samples.

\section{Statistical Analysis}

All analysis was performed using SPSS 23.0 (IBM). As specified a priori in our published protocol (22), primary contrasts were examined using a 2-way group x time mixed model analysis of variance (ANOVA), with experimental condition (group) as a betweensubjects factor and either pre-post visit or control/monitoring-intervention phase (time) as a within-subjects factor. Where the time-course of postprandial responses were quantified pre- and post-intervention, a three-way ANOVA was utilized to include timepoint in the model (group $\mathrm{x}$ time $\mathrm{x}$ timepoint). Wherever there were multiple contrasts for such timeseries data, the Greenhouse-Geisser correction was adopted for epsilon $<0.75$ and the Huynh-Feldt correction adopted for less severe asphericity. This parametric approach was applied irrespective of the distribution of data given that the type I error rate of the model is typically close to the nominal value $(p \leq 0.05)$ even when data are non-normally distributed $(62, p .109)$. Any relevant main 


\section{Science \\ Translational \\ Medicine}

effects of treatment or interaction effects were followed up using appropriate post-hoc tests to identify the location of variance, with a Ryan-Holm-Bonferroni stepwise correction to adjust the resulting $p$-values for multiple comparisons and thus avoid inflation of the type I error rate (63). Statistical significance was accepted at $p \leq 0.05$ and all data are reported as means \pm standard deviations and standard errors in tables and figures, respectively, with delta change scores (with $95 \%$ confidence intervals). 


\section{Supplementary Materials}

Figure S1. CONSORT flow diagram illustrating progress through each phase of the trial.

Figure S2. Schematic of the 8-week study design (A) and laboratory sessions 2 and 3 (B).

Table S1. Participant anthropometric and blood plasma characteristics at baseline. Data are means \pm standard deviation.

Table S2. CONSORT reporting checklist

Data file S1. Primary data. (Excel)

\section{References and Notes:}

1. I. Templeman, J. T. Gonzalez, D. Thompson, J. A. Betts, The role of intermittent fasting and meal timing in weight management and metabolic health. Proc. Nutr. Soc. 79, 76-87 (2020).

2. T. Ruge, L. Hodson, J. Cheeseman, A. L. Dennis, B. A. Fielding, S. M. Humphreys, K. N. Frayn, F. Karpe, Fasted to fed trafficking of Fatty acids in human adipose tissue reveals a novel regulatory step for enhanced fat storage. J. Clin. Endocrinol. Metab. 94, 1781-1788 (2009).

3. S. E. McQuaid, L. Hodson, M. J. Neville, A. L. Dennis, J. Cheeseman, S. M. Humphreys, T. Ruge, M. Gilbert, B. A. Fielding, K. N. Frayn, F. Karpe, Downregulation of adipose tissue fatty acid trafficking in obesity: a driver for ectopic fat deposition? Diabetes 60, 47-55 (2011).

4. R. de Cabo, M. P. Mattson, Effects of Intermittent Fasting on Health, Aging, and Disease. N. Engl. J. Med. 381, 2541-2551 (2019).

5. K. Tsintzas, K. Jewell, M. Kamran, D. Laithwaite, T. Boonsong, J. Littlewood, I. Macdonald, A. Bennett, Differential regulation of metabolic genes in skeletal muscle during starvation and refeeding in humans. $J$. Physiol. (Lond). 575, 291-303 (2006).

6. H. Jamshed, R. A. Beyl, D. L. Della Manna, E. S. Yang, E. Ravussin, C. M. Peterson, Early TimeRestricted Feeding Improves 24-Hour Glucose Levels and Affects Markers of the Circadian Clock, Aging, and Autophagy in Humans. Nutrients 11, (2019).

7. K. L. Ellacott, G. J. Morton, S. C. Woods, P. Tso, M. W. Schwartz, Assessment of feeding behavior in laboratory mice. Cell metabolism 12, 10-17 (2010).

8. Y. M. Roman, M. C. Dominguez, T. M. Easow, V. Pasupuleti, C. M. White, A. V. Hernandez, Effects of intermittent versus continuous dieting on weight and body composition in obese and overweight people: a systematic review and meta-analysis of randomized controlled trials. Int J Obes (Lond) 43, 2017-2027 (2019).

9. C. A. Rynders, E. A. Thomas, A. Zaman, Z. Pan, V. A. Catenacci, E. L. Melanson, Effectiveness of Intermittent Fasting and Time-Restricted Feeding Compared to Continuous Energy Restriction for Weight Loss. Nutrients 11, (2019).

10. I. Cioffi, A. Evangelista, V. Ponzo, G. Ciccone, L. Soldati, L. Santarpia, F. Contaldo, F. Pasanisi, E. Ghigo, S. Bo, Intermittent versus continuous energy restriction on weight loss and cardiometabolic outcomes: a systematic review and meta-analysis of randomized controlled trials. J Transl Med 16, 371 (2018).

11. S. D. Anton, K. Moehl, W. T. Donahoo, K. Marosi, S. A. Lee, A. G. Mainous, 3rd, C. Leeuwenburgh, M. P. Mattson, Flipping the Metabolic Switch: Understanding and Applying the Health Benefits of Fasting. Obesity 26, 254-268 (2018).

12. G. F. Cahill, Jr., Starvation in man. N. Engl. J. Med. 282, 668-675 (1970). 
13. M. R. Soeters, P. B. Soeters, M. G. Schooneman, S. M. Houten, J. A. Romijn, Adaptive reciprocity of lipid and glucose metabolism in human short-term starvation. Am J Physiol Endocrinol Metab 303, E1397-1407 (2012).

14. A. P. Koutnik, A. M. Poff, N. P. Ward, J. M. DeBlasi, M. A. Soliven, M. A. Romero, P. A. Roberson, C. D. Fox, M. D. Roberts, D. P. D'Agostino, Ketone Bodies Attenuate Wasting in Models of Atrophy. J Cachexia Sarcopenia Muscle, (2020).

15. E. F. Sutton, R. Beyl, K. S. Early, W. T. Cefalu, E. Ravussin, C. M. Peterson, Early Time-Restricted Feeding Improves Insulin Sensitivity, Blood Pressure, and Oxidative Stress Even without Weight Loss in Men with Prediabetes. Cell metabolism 27, 1212-1221 e1213 (2018).

16. R. Jones, P. Pabla, J. Mallinson, A. Nixon, T. Taylor, A. Bennett, K. Tsintzas, Two weeks of early timerestricted feeding (eTRF) improves skeletal muscle insulin and anabolic sensitivity in healthy men. Am. J. Clin. Nutr. 112, 1015-1028 (2020).

17. K. C. Allison, C. M. Hopkins, M. Ruggieri, A. M. Spaeth, R. S. Ahima, Z. Zhang, D. M. Taylor, N. Goel, Prolonged, Controlled Daytime versus Delayed Eating Impacts Weight and Metabolism. Curr. Biol., (2020).

18. S. Stekovic, S. J. Hofer, N. Tripolt, M. A. Aon, P. Royer, L. Pein, J. T. Stadler, T. Pendl, B. Prietl, J. Url, S. Schroeder, J. Tadic, T. Eisenberg, C. Magnes, M. Stumpe, E. Zuegner, N. Bordag, R. Riedl, A. Schmidt, E. Kolesnik, N. Verheyen, A. Springer, T. Madl, F. Sinner, R. de Cabo, G. Kroemer, B. Obermayer-Pietsch, J. Dengjel, H. Sourij, T. R. Pieber, F. Madeo, Alternate Day Fasting Improves Physiological and Molecular Markers of Aging in Healthy, Non-obese Humans. Cell metabolism 30, 462-476 e466 (2019).

19. J. F. Trepanowski, C. M. Kroeger, A. Barnosky, M. C. Klempel, S. Bhutani, K. K. Hoddy, K. Gabel, S. Freels, J. Rigdon, J. Rood, E. Ravussin, K. A. Varady, Effect of Alternate-Day Fasting on Weight Loss, Weight Maintenance, and Cardioprotection Among Metabolically Healthy Obese Adults: A Randomized Clinical Trial. JAMA internal medicine 177, 930-938 (2017).

20. K. Beaulieu, N. Casanova, P. Oustric, J. Turicchi, C. Gibbons, M. Hopkins, K. Varady, J. Blundell, G. Finlayson, Matched Weight Loss Through Intermittent or Continuous Energy Restriction Does Not Lead To Compensatory Increases in Appetite and Eating Behavior in a Randomized Controlled Trial in Women with Overweight and Obesity. J. Nutr. 150, 623-633 (2020).

21. A. T. Hutchison, B. Liu, R. E. Wood, A. D. Vincent, C. H. Thompson, N. J. O'Callaghan, G. A. Wittert, L. K. Heilbronn, Effects of Intermittent Versus Continuous Energy Intakes on Insulin Sensitivity and Metabolic Risk in Women with Overweight. Obesity 27, 50-58 (2019).

22. I. Templeman, D. Thompson, J. Gonzalez, J. P. Walhin, S. Reeves, P. J. Rogers, J. M. Brunstrom, L. G. Karagounis, K. Tsintzas, J. A. Betts, Intermittent fasting, energy balance and associated health outcomes in adults: study protocol for a randomised controlled trial. Trials 19, 86 (2018).

23. K. A. Varady, Intermittent versus daily calorie restriction: which diet regimen is more effective for weight loss? Obesity reviews : an official journal of the International Association for the Study of Obesity 12, e593-601 (2011).

24. B. A. Alhamdan, A. Garcia-Alvarez, A. H. Alzahrnai, J. Karanxha, D. R. Stretchberry, K. J. Contrera, A. F. Utria, L. J. Cheskin, Alternate-day versus daily energy restriction diets: which is more effective for weight loss? A systematic review and meta-analysis. Obes Sci Pract 2, 293-302 (2016).

25. M. H. Vendelbo, A. B. Moller, B. Christensen, B. Nellemann, B. F. Clasen, K. S. Nair, J. O. Jorgensen, N. Jessen, N. Moller, Fasting Increases Human Skeletal Muscle Net Phenylalanine Release and This Is Associated with Decreased mTOR Signaling. PLoS ONE 9, e102031 (2014).

26. J. F. Trepanowski, C. M. Kroeger, A. Barnosky, M. Klempel, S. Bhutani, K. K. Hoddy, J. Rood, E. Ravussin, K. A. Varady, Effects of alternate-day fasting or daily calorie restriction on body composition, fat distribution, and circulating adipokines: Secondary analysis of a randomized controlled trial. Clin. Nutr. 37, 1871-1878 (2018).

27. S. Cienfuegos, K. Gabel, F. Kalam, M. Ezpeleta, E. Wiseman, V. Pavlou, S. Lin, M. L. Oliveira, K. A. Varady, Effects of 4- and 6-h Time-Restricted Feeding on Weight and Cardiometabolic Health: A Randomized Controlled Trial in Adults with Obesity. Cell metabolism 32, 366-378 e363 (2020).

28. T. Abe, S. J. Dankel, J. P. Loenneke, Body Fat Loss Automatically Reduces Lean Mass by Changing the Fat-Free Component of Adipose Tissue. Obesity 27, 357-358 (2019).

29. D. A. Lowe, N. Wu, L. Rohdin-Bibby, A. H. Moore, N. Kelly, Y. E. Liu, E. Philip, E. Vittinghoff, S. B. Heymsfield, J. E. Olgin, J. A. Shepherd, E. J. Weiss, Effects of Time-Restricted Eating on Weight Loss and Other Metabolic Parameters in Women and Men With Overweight and Obesity: The TREAT Randomized Clinical Trial. JAMA internal medicine, (2020). 
30. C. S. Fox, J. M. Massaro, U. Hoffmann, K. M. Pou, P. Maurovich-Horvat, C. Y. Liu, R. S. Vasan, J. M. Murabito, J. B. Meigs, L. A. Cupples, R. B. D'Agostino, Sr., C. J. O'Donnell, Abdominal visceral and subcutaneous adipose tissue compartments: association with metabolic risk factors in the Framingham Heart Study. Circulation 116, 39-48 (2007).

31. T. M. Sundfor, M. Svendsen, S. Tonstad, Effect of intermittent versus continuous energy restriction on weight loss, maintenance and cardiometabolic risk: A randomized 1-year trial. Nutrition, metabolism, and cardiovascular diseases : NMCD 28, 698-706 (2018).

32. M. C. Klempel, S. Bhutani, M. Fitzgibbon, S. Freels, K. A. Varady, Dietary and physical activity adaptations to alternate day modified fasting: implications for optimal weight loss. Nutr J 9, 35 (2010).

33. L. M. Redman, L. K. Heilbronn, C. K. Martin, L. de Jonge, D. A. Williamson, J. P. Delany, E. Ravussin, C. T. Pennington, Metabolic and behavioral compensations in response to caloric restriction: implications for the maintenance of weight loss. PLoS ONE 4, e4377 (2009).

34. J. A. Betts, J. D. Richardson, E. A. Chowdhury, G. D. Holman, K. Tsintzas, D. Thompson, The causal role of breakfast in energy balance and health: a randomized controlled trial in lean adults. Am. J. Clin. Nutr. 100, 539-547 (2014).

35. E. A. Chowdhury, J. D. Richardson, G. D. Holman, K. Tsintzas, D. Thompson, J. A. Betts, The causal role of breakfast in energy balance and health: a randomized controlled trial in obese adults. Am. J. Clin. Nutr. 103, 747-756 (2016).

36. B. E. Ainsworth, W. L. Haskell, S. D. Herrmann, N. Meckes, D. R. Bassett, Jr., C. Tudor-Locke, J. L. Greer, J. Vezina, M. C. Whitt-Glover, A. S. Leon, 2011 Compendium of Physical Activities: a second update of codes and MET values. Med Sci Sports Exerc 43, 1575-1581 (2011).

37. A. Hengist, R. M. Edinburgh, R. G. Davies, J. P. Walhin, J. Buniam, L. J. James, P. J. Rogers, J. T. Gonzalez, J. A. Betts, Physiological responses to maximal eating in men. Br. J. Nutr., 1-11 (2020).

38. J. P. Walhin, J. D. Richardson, J. A. Betts, D. Thompson, Exercise counteracts the effects of short-term overfeeding and reduced physical activity independent of energy imbalance in healthy young men. The Journal of physiology 591, 6231-6243 (2013).

39. R. James, L. J. James, D. J. Clayton, Anticipation of 24 h severe energy restriction increases energy intake and reduces physical activity energy expenditure in the prior $24 \mathrm{~h}$, in healthy males. Appetite 152, 104719 (2020).

40. A. Borai, C. Livingstone, I. Kaddam, G. Ferns, Selection of the appropriate method for the assessment of insulin resistance. BMC Med Res Methodol 11, 158 (2011).

41. B. G. Nordestgaard, A Test in Context: Lipid Profile, Fasting Versus Nonfasting. J. Am. Coll. Cardiol. 70, 1637-1646 (2017).

42. A. T. Hutchison, P. Regmi, E. N. C. Manoogian, J. G. Fleischer, G. A. Wittert, S. Panda, L. K. Heilbronn, Time-Restricted Feeding Improves Glucose Tolerance in Men at Risk for Type 2 Diabetes: A Randomized Crossover Trial. Obesity 27, 724-732 (2019).

43. R. Antoni, K. L. Johnston, A. L. Collins, M. D. Robertson, Intermittent v. continuous energy restriction: differential effects on postprandial glucose and lipid metabolism following matched weight loss in overweight/obese participants. Br. J. Nutr. 119, 507-516 (2018).

44. S. Stekovic, S. J. Hofer, N. Tripolt, M. A. Aon, P. Royer, L. Pein, J. T. Stadler, T. Pendl, B. Prietl, J. Url, S. Schroeder, J. Tadic, T. Eisenberg, C. Magnes, M. Stumpe, E. Zuegner, N. Bordag, R. Riedl, A. Schmidt, E. Kolesnik, N. Verheyen, A. Springer, T. Madl, F. Sinner, R. de Cabo, G. Kroemer, B. Obermayer-Pietsch, J. Dengjel, H. Sourij, T. R. Pieber, F. Madeo, Alternate Day Fasting Improves Physiological and Molecular Markers of Aging in Healthy, Non-obese Humans. Cell metabolism 31, 878-881 (2020).

45. L. M. Sparks, L. M. Redman, K. E. Conley, M. E. Harper, F. Yi, A. Hodges, A. Eroshkin, S. R. Costford, M. E. Gabriel, C. Shook, H. H. Cornnell, E. Ravussin, S. R. Smith, Effects of 12 Months of Caloric Restriction on Muscle Mitochondrial Function in Healthy Individuals. J. Clin. Endocrinol. Metab. 102, 111-121 (2017).

46. A. Oswal, G. Yeo, Leptin and the control of body weight: a review of its diverse central targets, signaling mechanisms, and role in the pathogenesis of obesity. Obesity 18, 221-229 (2010).

47. R. S. Ahima, D. Prabakaran, C. Mantzoros, D. Qu, B. Lowell, E. Maratos-Flier, J. S. Flier, Role of leptin in the neuroendocrine response to fasting. Nature 382, 250-252 (1996).

48. C. A. Matson, M. F. Wiater, J. L. Kuijper, D. S. Weigle, Synergy between leptin and cholecystokinin (CCK) to control daily caloric intake. Peptides 18, 1275-1278 (1997).

49. S. Guilmeau, M. Buyse, A. Tsocas, J. P. Laigneau, A. Bado, Duodenal leptin stimulates cholecystokinin secretion: evidence of a positive leptin-cholecystokinin feedback loop. Diabetes 52, 1664-1672 (2003). 
50. A. Barnosky, C. M. Kroeger, J. F. Trepanowski, M. C. Klempel, S. Bhutani, K. K. Hoddy, K. Gabel, S. A. Shapses, K. A. Varady, Effect of alternate day fasting on markers of bone metabolism: An exploratory analysis of a 6-month randomized controlled trial. Nutr Healthy Aging 4, 255-263 (2017).

51. I. A. Heikura, L. M. Burke, J. A. Hawley, M. L. Ross, L. Garvican-Lewis, A. P. Sharma, A. K. A. McKay, J. J. Leckey, M. Welvaert, L. McCall, K. E. Ackerman, A Short-Term Ketogenic Diet Impairs Markers of Bone Health in Response to Exercise. Front Endocrinol (Lausanne) 10, 880 (2019).

52. L. S. Lundell, E. B. Parr, B. L. Devlin, L. R. Ingerslev, A. Altintas, S. Sato, P. Sassone-Corsi, R. Barres, J. R. Zierath, J. A. Hawley, Time-restricted feeding alters lipid and amino acid metabolite rhythmicity without perturbing clock gene expression. Nat Commun 11, 4643 (2020).

53. P. Lewis, H. Oster, H. W. Korf, R. G. Foster, T. C. Erren, Food as a circadian time cue - evidence from human studies. Nat Rev Endocrinol 16, 213-223 (2020).

54. L. Perrin, U. Loizides-Mangold, S. Chanon, C. Gobet, N. Hulo, L. Isenegger, B. D. Weger, E. Migliavacca, A. Charpagne, J. A. Betts, J. P. Walhin, I. Templeman, K. Stokes, D. Thompson, K. Tsintzas, M. Robert, C. Howald, H. Riezman, J. N. Feige, L. G. Karagounis, J. D. Johnston, E. T. Dermitzakis, F. Gachon, E. Lefai, C. Dibner, Transcriptomic analyses reveal rhythmic and CLOCK-driven pathways in human skeletal muscle. eLife 7, (2018).

55. U. Loizides-Mangold, L. Perrin, B. Vandereycken, J. A. Betts, J. P. Walhin, I. Templeman, S. Chanon, B. D. Weger, C. Durand, M. Robert, J. Paz Montoya, M. Moniatte, L. G. Karagounis, J. D. Johnston, F. Gachon, E. Lefai, H. Riezman, C. Dibner, Lipidomics reveals diurnal lipid oscillations in human skeletal muscle persisting in cellular myotubes cultured in vitro. Proc. Natl. Acad. Sci. U. S. A. 114, E8565-E8574 (2017).

56. A. L. Friedlander, B. Braun, M. Pollack, J. R. MacDonald, C. S. Fulco, S. R. Muza, P. B. Rock, G. C. Henderson, M. A. Horning, G. A. Brooks, A. R. Hoffman, A. Cymerman, Three weeks of caloric restriction alters protein metabolism in normal-weight, young men. Am J Physiol Endocrinol Metab 289, E446-455 (2005).

57. A. Molfino, A. Cascino, C. Conte, C. Ramaccini, F. Rossi Fanelli, A. Laviano, Caloric restriction and Lcarnitine administration improves insulin sensitivity in patients with impaired glucose metabolism. JPEN. J. Parenter. Enteral Nutr. 34, 295-299 (2010).

58. K. R. Westerterp, Diet induced thermogenesis. Nutrition \& Metabolism (Lond) 1, 5 (2004).

59. K. N. Frayn, Calculation of substrate oxidation rates in vivo from gaseous exchange. J. Appl. Physiol. 55, 628-634 (1983).

60. C. Compher, D. Frankenfield, N. Keim, L. Roth-Yousey, Best practice methods to apply to measurement of resting metabolic rate in adults: a systematic review. J. Am. Diet. Assoc. 106, 881-903 (2006).

61. K. Tsintzas, L. Norton, K. Chokkalingam, N. Nizamani, S. Cooper, F. Stephens, R. Billeter, A. Bennett, Independent and combined effects of acute physiological hyperglycaemia and hyperinsulinaemia on metabolic gene expression in human skeletal muscle. Clin Sci (Lond) 124, 675-684 (2013).

62. S. E. Maxwell, H. D. Delaney, Designing Experiments and Analyzing Data: a model comparison perspective. (Wadsworth, Belmont, 1990).

63. J. Ludbrook, Multiple comparison procedures updated. Clin. Exp. Pharmacol. Physiol. 25, 1032-1037 (1998).

64. J. C. Levy, D. R. Matthews, M. P. Hermans, Correct homeostasis model assessment (HOMA) evaluation uses the computer program. Diabetes Care 21, 2191-2192 (1998).

Acknowledgments: The authors thank all those who volunteered to take part in this research and

Isobel Townsend for collating raw data for archiving. Funding: This work was funded by the University of Bath and Nottingham Trent University. Author contributions: IT, LK, KT, DT, JAB contributed to conceptualization; IT, HAS, YCC, JAB curated data; IT, HAS, EC, YCC, 
AH, RS, DC, IV, SR, AW, JPW, JAB analyzed data; IT, DC, IV, DT, JAB contributed to funding acquisition; IT, HAS, YCC, HC, DJ-B, JPW, DT, JAB conducted the investigation; IT, EC, LK, KT, JPW, JTG, DT, JAB contributed to methodology; IT, JTG, DT, JAB contributed to project administration; JTG, DT, JAB provided resources and supervision; JC, SR provided software; HAS, EC, RS, JPW, DT, JAB were involved in validation; IT, HAS, JTG, JAB contributed to data visualization; IT, JAB write the original draft; all authors contributed to review/editing and approved the final submission. Competing interests: YCC has received research funding from Ministry of Science and Technology in Taiwan. HC has received research funding from ESRC, European Hydration Institute, and the Esther Olssons stiftelse II \& Anna Jönssons Minnesfond; has conducted research for Tate \& Lyle; and has received conference, travel and accommodation fees from Danone Nutritia Research. DC has received research funding from the British Nutrition Foundation. LK is an employee of Nestlé. KT has received research funding from BBSRC, MRC and Nestlé. SR has received research funding from Kellogg's, the Organix Foundation and the MRC Newton-Ungku Omar Fund. JTG has received research funding from Arla Foods Ingredients, Lucozade Ribena Suntory, Kenniscentrum Suiker and Voeding, and PepsiCo. DT has received research funding from Unilever. JAB is a named investigator on research grants funded by BBSRC, MRC, British Heart Foundation, Rare Disease Foundation, EU Hydration Institute, GlaxoSmithKline, Nestlé, Lucozade Ribena Suntory, ARLA foods and Kennis Centrum Suiker; has completed paid consultancy for PepsiCo, Kellogg's and SVGC; receives an annual stipend as Editor-in Chief of International Journal of Sport Nutrition \& Exercise Metabolism; and receives an annual honorarium as a member of the academic advisory board for the International Olympic Committee Diploma in Sports Nutrition. Data and 
materials availability: All data associated with this paper is present in main text or supplementary materials. Primary data are available in data file S1.

\section{Figure Captions:}

Figure 1: Changes in body composition and components of energy balance at baseline and during interventions involving either: $25 \%$ daily energy restriction diet (75:75); alternate-day fasting with $50 \%$ refeeding for a net $25 \%$ energy restriction diet $(\mathbf{0 : 1 5 0})$; or alternate-day fasting with $100 \%$ refeeding for a net 0\% energy restriction diet (0:200). A) Fat and fat-free mass. B) Energy intake C) Energy expenditure D) Post-prandial substrate oxidation. E) Physical activity thermogenesis. F) Changes in physical activity thermogenesis from baseline. $p$-values are post-hoc between group differences in response [panel $\mathbf{A}$ ] and post-hoc within group responses [panels $\mathbf{B}$ and $\mathbf{C}$ ]. Data are means and standard error of the mean (all conditions $n=12$ ).

Figure 2: Plasma metabolite concentrations in response to breakfast and lunch before and after following interventions involving either: $25 \%$ daily energy restriction diet (75:75); alternate-day fasting with $50 \%$ refeeding for a net $25 \%$ energy restriction diet (0:150); or alternate-day fasting with $100 \%$ refeeding for a net $0 \%$ energy restriction diet (0:200). A) Glucose. B) Non-esterified fatty acids (NEFA). C) Triacylglycerol. D) Glycerol. Data are means and standard error of the mean (all conditions $\mathrm{n}=12$ ).

Figure 3: Plasma hormone and telopeptide concentrations in response to breakfast and lunch before and after interventions involving either: a 25\% daily energy restriction diet (75:75); alternate-day fasting with $50 \%$ refeeding for a net $25 \%$ energy restriction diet (0:150); or alternate-day fasting with $100 \%$ refeeding for a net 0\% energy restriction diet (0:200). A) Insulin $(\mathrm{n}=12 / 12 / 12)$. B) Carboxy-terminal telopeptide of type I collagen crosslinks (CTX; $n=11 / 9 / 10)$. 
C) Acylated ghrelin (n=11/9/10). D) Peptide yy (tyrosine-tyrosine; $n=10 / 7 / 7)$. Data are means and standard error of the mean.

Figure 4: Relative changes in adipose tissue mRNA expression before and after interventions involving either: $25 \%$ daily energy restriction diet (75:75); alternate-day fasting with 50\% refeeding for a net $25 \%$ energy restriction diet $(\mathbf{0 : 1 5 0})$; or alternate-day fasting with $100 \%$ refeeding for a net $0 \%$ energy restriction diet $(\mathbf{0 : 2 0 0})$. The colour hue and intensity represent the direction and effect size (Cohen's $d$ ), respectively, for the pre- to post-intervention change within each group $(n=8 / 6 / 4)$. Genes with a group $x$ time interaction $p \leq 0.05$ are in bold; $p$-values are post-hoc within group responses.

\section{Table Captions:}

Table 1. Body composition responses pre- and post-intervention. Data are means \pm standard deviation, delta change (95\% confidence intervals) for within-group responses and $p$-values for between-group differences in response.

Table 2. Blood plasma responses pre- and post-intervention. Data are means \pm standard deviation and delta change (95\% confidence intervals) for within-group responses and $p$-values for between-group differences in response. 
Table 1. Body composition responses pre- to post-intervention. Data are means \pm standard deviation, delta change (95\% confidence intervals) for within-group responses and $p$-values for between-group differences in response.

\begin{tabular}{|c|c|c|c|c|c|c|c|c|c|}
\hline & \multirow{2}{*}{\multicolumn{2}{|c|}{$\begin{array}{c}\text { Daily Energy Restriction } \\
\text { (ER; 75:75) } \\
\end{array}$}} & \multirow{2}{*}{\multicolumn{2}{|c|}{$\begin{array}{c}\text { Alternate-Day Fasting w. net ER } \\
(0: 150)\end{array}$}} & \multirow{2}{*}{\multicolumn{2}{|c|}{$\begin{array}{c}\text { Alternate-day Fasting w/o net ER } \\
(0: 200)\end{array}$}} & \multicolumn{3}{|c|}{$p$-value } \\
\hline & & & & & & & \multirow{2}{*}{$\begin{array}{l}75: 75 \\
v S \\
0: 150\end{array}$} & \multirow{2}{*}{$\begin{array}{l}0: 150 \\
v S \\
0: 200\end{array}$} & \multirow{2}{*}{$\begin{array}{l}75: 75 \\
v S \\
0: 200\end{array}$} \\
\hline & Pre & $\Delta$ post & Pre & $\Delta$ post & Pre & $\Delta$ post & & & \\
\hline Body Mass (kg) ${ }^{\mathbf{a}}$ & $72.1 \pm 10.2$ & $-1.91(-1.29,-2.54)$ & $72.3 \pm 8.2$ & $-1.60(-0.93,-2.28)$ & $67.7 \pm 7.8$ & $-0.52(0.18,-1.21)$ & 0.46 & 0.04 & 0.01 \\
\hline Body Mass Index $\left(\mathrm{kg} \cdot \mathrm{m}^{-2}\right)^{\mathbf{a}}$ & $24.0 \pm 1.9$ & $-0.6(-0.5,-0.8)$ & $24.0 \pm 2.3$ & $-0.5(-0.3,-0.8)$ & $23.7 \pm 2.1$ & $-0.2(0.1,-0.4)$ & 0.45 & $\mathbf{0 . 0 3}$ & 0.003 \\
\hline Fat Mass $(\mathrm{kg})^{\mathbf{b}}$ & $18.3 \pm 4.1$ & $-1.75(-1.25,-2.25)$ & $15.9 \pm 5.2$ & $-0.74(-0.02,-1.24)$ & $17.1 \pm 6.7$ & $-0.12(0.31,-0.55)$ & 0.01 & 0.05 & 0.0001 \\
\hline Fat Mass Index $\left(\mathrm{kg} \cdot \mathrm{m}^{-2}\right)^{\mathbf{b}}$ & $6.2 \pm 1.5$ & $-0.59(-0.42,-0.76)$ & $5.4 \pm 2.2$ & $-0.24(-0.08,-0.41)$ & $6.0 \pm 2.4$ & $-0.01(0.11,-0.20)$ & 0.01 & 0.06 & 0.0001 \\
\hline Percent Body Fat $(\%)^{\mathbf{b}}$ & $25.7 \pm 6.2$ & $-1.81(-1.25,-2.37)$ & $22.4 \pm 7.9$ & $-0.56(0.12,-1.24)$ & $25.3 \pm 9.1$ & $0.10(-0.45,0.66)$ & 0.02 & 0.21 & 0.001 \\
\hline Waist Circumference $(\mathrm{cm})$ & $84.0 \pm 7.1$ & $-2.4(-1.3,-3.6)$ & $83.2 \pm 4.6$ & $-1.7(-0.5,-2.8)$ & $80.0 \pm 7.9$ & $-0.7(0.6,-1.9)$ & 0.42 & 0.42 & 0.09 \\
\hline Visceral Fat Mass (g) & $383 \pm 164$ & $-31(-12,-51)$ & $356 \pm 95$ & $-34(-13,-56)$ & $311 \pm 162$ & $-8(33,-48)$ & 0.81 & 0.65 & 0.65 \\
\hline Fat-Free Mass (kg) & $53.0 \pm 10.3$ & $-0.03(0.40,-0.47)$ & $55.4 \pm 9.8$ & $-0.75(0.09,-1.59)$ & $49.5 \pm 8.8$ & $-0.53(0.004,-1.06)$ & 0.35 & 0.64 & 0.35 \\
\hline Lean Soft Tissue Mass (kg) & $50.3 \pm 10.0$ & $-0.03(0.40,-0.45)$ & $52.6 \pm 9.4$ & $-0.74(0.09,-1.57)$ & $47.0 \pm 8.4$ & $-0.52(0.01,-1.06)$ & 0.33 & 0.33 & 0.63 \\
\hline Bone Mineral Content $(\mathrm{g})$ & $2676 \pm 431$ & $-8.5(30.8,-47.8)$ & $2747 \pm 453$ & $-6.6(26.3,-39.4)$ & $2499 \pm 428$ & $-5.9(18.9,-30.8)$ & $>0.99$ & $>0.99$ & $>0.99$ \\
\hline Bone Mineral Density $\left(\mathrm{g} \cdot \mathrm{cm}^{-3}\right)$ & $1.20 \pm 0.10$ & $-0.004(0.01,-0.18)$ & $1.23 \pm 0.11$ & $-0.01(0.01,-0.02)$ & $1.18 \pm 0.10$ & $-0.01(-0.004,-0.03)$ & $>0.99$ & $>0.99$ & $>0.99$ \\
\hline
\end{tabular}

a denotes significant group $\mathrm{x}$ time (pre-post) interactions at $p=0.01$

b denotes significant group $\mathrm{x}$ time (pre-post) interactions at $p=0.0001$

Within-group responses where the confidence interval does not include zero are in bold text; between group $p$-values are independent $t$-tests, adjusted for multiple comparisons. 
(64)

Table 2. Blood plasma responses pre- to post-intervention. Data are means \pm standard deviation and delta change (95\% confidence intervals) for within-group responses and $p$-values for between-group differences in response.

\begin{tabular}{|c|c|c|c|c|c|c|c|c|c|}
\hline & \multirow{2}{*}{\multicolumn{2}{|c|}{$\begin{array}{c}\text { Daily Energy Restriction } \\
\text { (ER; 75:75) }\end{array}$}} & \multirow{2}{*}{\multicolumn{2}{|c|}{$\begin{array}{c}\text { Alternate-Day Fasting w. net ER } \\
(0: 150)\end{array}$}} & \multirow{2}{*}{\multicolumn{2}{|c|}{$\begin{array}{c}\text { Alternate-day Fasting w/o net ER } \\
(0: 200)\end{array}$}} & \multicolumn{3}{|c|}{$p$-value } \\
\hline & & & & & & & \multirow{2}{*}{$\begin{array}{l}75: 75 \\
v s \\
0: 150\end{array}$} & \multirow{2}{*}{$\begin{array}{c}0: 150 \\
v S \\
0: 200\end{array}$} & \multirow{2}{*}{$\begin{aligned} 75: 75 \\
v s \\
0: 200\end{aligned}$} \\
\hline & Pre & $\Delta$ post & Pre & $\Delta$ post & Pre & $\Delta$ post & & & \\
\hline$\overline{\text { Glucose }\left(\mathrm{mmol} \cdot \boldsymbol{I}^{-1}\right)}$ & $5.27 \pm 0.38$ & $0.01(-0.13,0.15)$ & $5.62 \pm 0.37$ & $0.04(-0.29,0.38)$ & $5.14 \pm 0.67$ & $0.10(-0.20,0.40)$ & $>0.99$ & $>0.99$ & $>0.99$ \\
\hline iAUC $C^{\mathbf{a}} \mathrm{Meal1}\left(\mathrm{mmol} \cdot l^{-1} \cdot 180 \mathrm{~min}\right)$ & $136 \pm 103$ & $26.8(-29.9,83.4)$ & $106 \pm 68$ & $9.9(-75.1,94.9)$ & $90 \pm 30$ & $0.8(-22.8,24.5)$ & $>0.99$ & $>0.99$ & $>0.99$ \\
\hline iAUC $C^{\mathbf{a}}$ Meal2 $\left(\mathrm{mmol} \cdot \mathrm{l}^{-1} \cdot 120 \mathrm{~min}\right)$ & $197 \pm 65$ & $-1.8(26.7,-30.2)$ & $196 \pm 90$ & $1.5(-47.5,50.4)$ & $176 \pm 72$ & $-0.9(21.2,-23.0)$ & $>0.99$ & $>0.99$ & $>0.99$ \\
\hline Insulin (pmol·-1-1) & $20.6 \pm 8.2$ & $-3.5(1.0,-7.9)$ & $19.0 \pm 5.0$ & $-0.01(4.7,-4.7)$ & $18.5 \pm 5.8$ & $2.7(-2.1,7.5)$ & 0.52 & 0.52 & 0.16 \\
\hline iAUC $C^{\mathbf{a}} \mathrm{Meall}\left(\mathrm{nmol} \cdot l^{-1} \cdot 180 \mathrm{~min}\right)$ & $13.1 \pm 4.9$ & $1.0(-3.2,5.2)$ & $17.9 \pm 7.8$ & $2.5(-4.0,8.7)$ & $18.5 \pm 8.0$ & $0.3(-3.6,4.3)$ & $>0.99$ & $>0.99$ & $>0.99$ \\
\hline 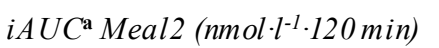 & $14.9 \pm 5.4$ & $-0.1(3.1,-3.3)$ & $16.7 \pm 6.0$ & $0.3(-1.2,1.9)$ & $20.2 \pm 11.3$ & $-0.5(5.1,-6.0)$ & $>0.99$ & $>0.99$ & $>0.99$ \\
\hline HOMA2-IR ${ }^{b}$ & $0.46 \pm 0.18$ & $-0.08(0.02,-0.17)$ & $0.43 \pm 0.11$ & $-0.004(0.10,-0.11)$ & $0.40 \pm 0.11$ & $0.06(-0.04,0.16)$ & 0.52 & 0.52 & 0.12 \\
\hline $\operatorname{NEFA}^{c}\left(\mathrm{mmol}^{-\mathrm{I}^{-1}}\right)$ & $0.48 \pm 0.34$ & $-0.02(0.08,-0.13)$ & $0.33 \pm 0.09$ & $0.01(-0.08,0.09)$ & $0.34 \pm 0.18$ & $-0.04(0.09,-0.16)$ & $>0.99$ & $>0.99$ & $>0.99$ \\
\hline$A U C^{\mathbf{a}}\left(\mathrm{mmol} \cdot l^{-1} \cdot 330 \mathrm{~min}\right)$ & $49.7 \pm 21.1$ & $-3.8(5.0,-12.7)$ & $39.7 \pm 10.7$ & $-0.1(6.6,-6.8)$ & $41.5 \pm 17.6$ & $-2.1(8.9,-13.0)$ & $>0.99$ & $>0.99$ & $>0.99$ \\
\hline Glycerol $\left(\mu \mathrm{mol} \cdot \mathrm{l}^{-1}\right)$ & $58.0 \pm 32.9$ & $-0.39(18.5,-19.3)$ & $40.6 \pm 29.9$ & $0.44(-16.0,16.9)$ & $35.9 \pm 24.5$ & $-1.75(14.7,-18.2)$ & $>0.99$ & $>0.99$ & $>0.99$ \\
\hline$A U C^{\mathbf{a}}\left(\mathrm{mmol} \cdot l^{-1} \cdot 330 \mathrm{~min}\right)$ & $12.3 \pm 5.0$ & $1.7(-0.8,4.2)$ & $10.7 \pm 4.1$ & $0.3(-1.1,1.7)$ & $10.7 \pm 4.1$ & $-0.1(1.3,-1.6)$ & 0.60 & 0.63 & 0.54 \\
\hline Triacylglycerol $\left(\mathrm{m} \mathrm{mol} \cdot \mathrm{l}^{-1}\right)$ & $0.81 \pm 0.32$ & $-0.04(0.13,-0.21)$ & $0.97 \pm 0.33$ & $-0.12(0.02,-0.27)$ & $0.92 \pm 0.32$ & $-0.02(0.13,-0.18)$ & $>0.99$ & $>0.99$ & $>0.99$ \\
\hline$A U C^{\mathrm{a}}\left(\mathrm{mmol} \cdot \mathrm{l}^{-1} \cdot 330 \mathrm{~min}\right)^{\mathrm{e}}$ & $236 \pm 109$ & $38.5(-3.1,80.2)$ & $384 \pm 126$ & $-46.7(1.1,-94.4)$ & $345 \pm 165$ & $-5.0(40.4,-50.4)$ & 0.02 & 0.27 & 0.27 \\
\hline Total Cholesterol $\left(\mathrm{mmol} \cdot \mathrm{l}^{-1}\right)$ & $4.75 \pm 0.97$ & $-0.28(-0.09,-0.48)$ & $4.74 \pm 0.77$ & $0.00(-0.23,0.23)$ & $4.68 \pm 0.96$ & $0.01(-0.24,0.27)$ & 0.16 & 0.94 & 0.16 \\
\hline HDL $^{\mathrm{d}}$ Cholesterol $\left(\mathrm{mmol}^{-1}\right)$ & $1.64 \pm 0.43$ & $-0.07(0.03,-0.18)$ & $1.53 \pm 0.43$ & $0.05(-0.04,0.14)$ & $1.60 \pm 0.41$ & $0.04(-0.05,0.13)$ & 0.21 & 0.91 & 0.21 \\
\hline LDL $^{\mathrm{d}}$ Cholesterol $\left(\mathrm{mmol}^{-1} \mathrm{l}^{-1}\right)$ & $2.96 \pm 0.93$ & $-0.24(-0.07,-0.41)$ & $3.06 \pm 0.85$ & $-0.10(0.12,-0.31)$ & $2.82 \pm 1.18$ & $-0.02(0.27,-0.31)$ & 0.55 & 0.66 & 0.53 \\
\hline Leptin $\left(\mu \mathrm{g} \cdot \mathrm{I}^{-1}\right)^{\mathrm{e}}$ & $9.3 \pm 6.0$ & $-3.8(-1.5,-6.1)$ & $10.5 \pm 13.9$ & $-2.8(0.4,-6.0)$ & $16.4 \pm 15.9$ & $0.2(-1.3,1.7)$ & 0.59 & 0.16 & 0.01 \\
\hline Adiponectin $\left(\mathbf{m g} \cdot \mathbf{l}^{-1}\right)$ & $9.9 \pm 2.6$ & $-0.7(-0.3,-1.2)$ & $8.8 \pm 2.6$ & $-0.3(0.3,-0.6)$ & $10.5 \pm 4.9$ & $-0.2(1.0,-1.5)$ & 0.21 & 0.97 & 0.87 \\
\hline
\end{tabular}

a iAUC $=$ incremental area under the curve from concentrations prior to each Meal (i.e. Breakfast and Lunch); AUC $=$ total area under the curve across both meals

b HOMA-IR = Homeostatic Model Assessment of Insulin Resistance 2 (64)

c NEFA = Non-Esterified Fatty Acids

d HDL/LDL = High-/Low-Den sity Lipoprotein

e denotes significant group $\mathrm{x}$ time (pre-post) interactions at $p \leq 0.05$

Within-group responses where the confidence interval does not include zero are in bold text; between group $p$-values are independent $t$-tests, adjusted for multiple comparisons. 
A

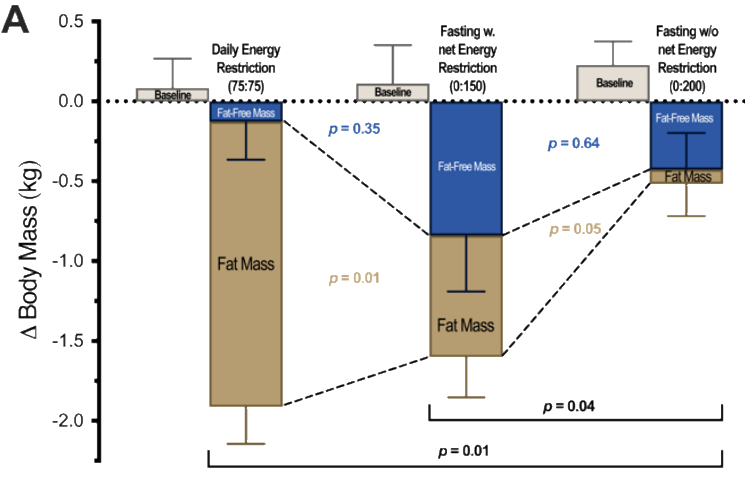

C
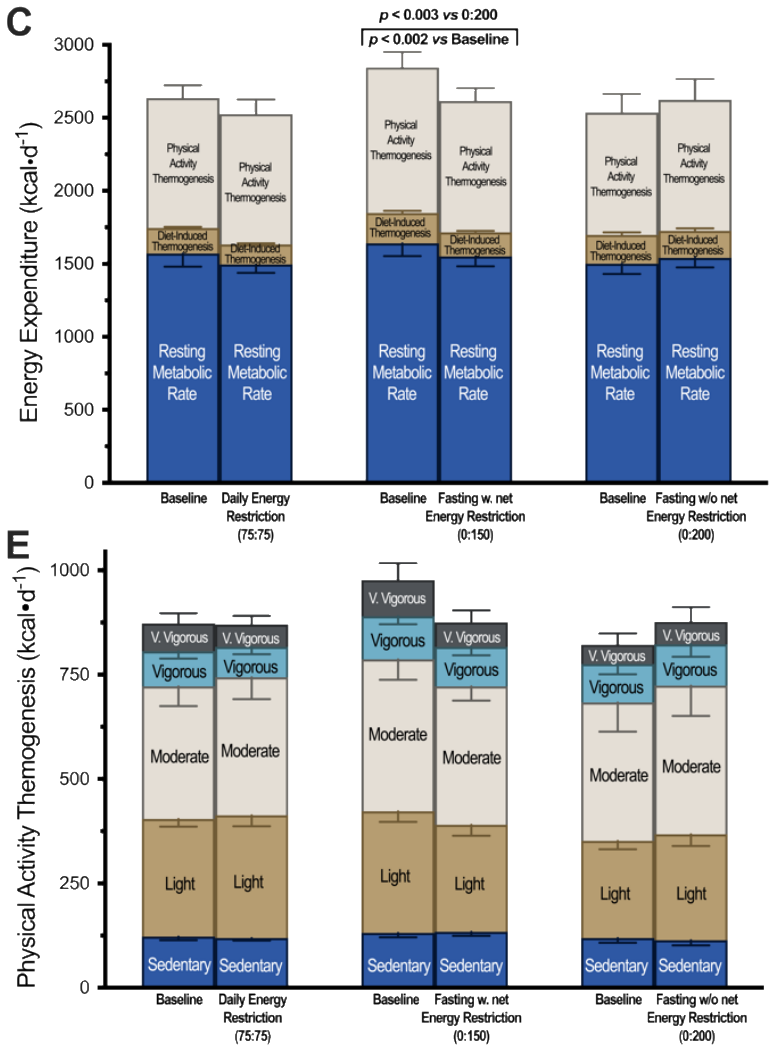

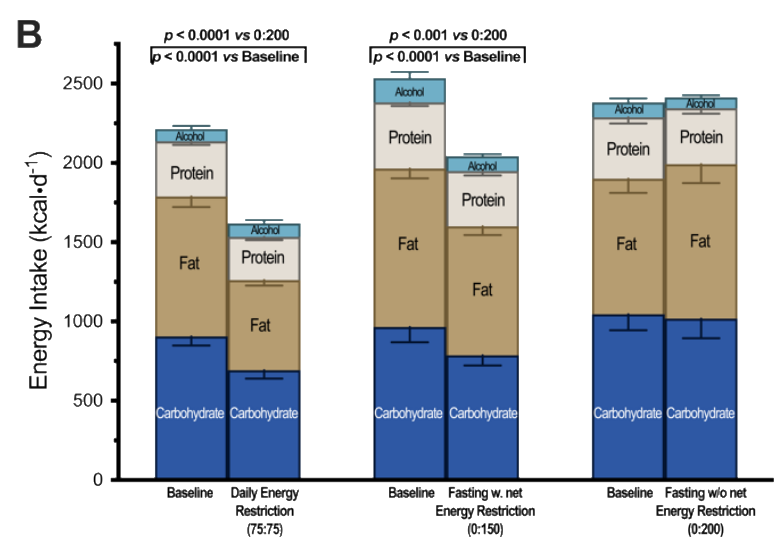

D
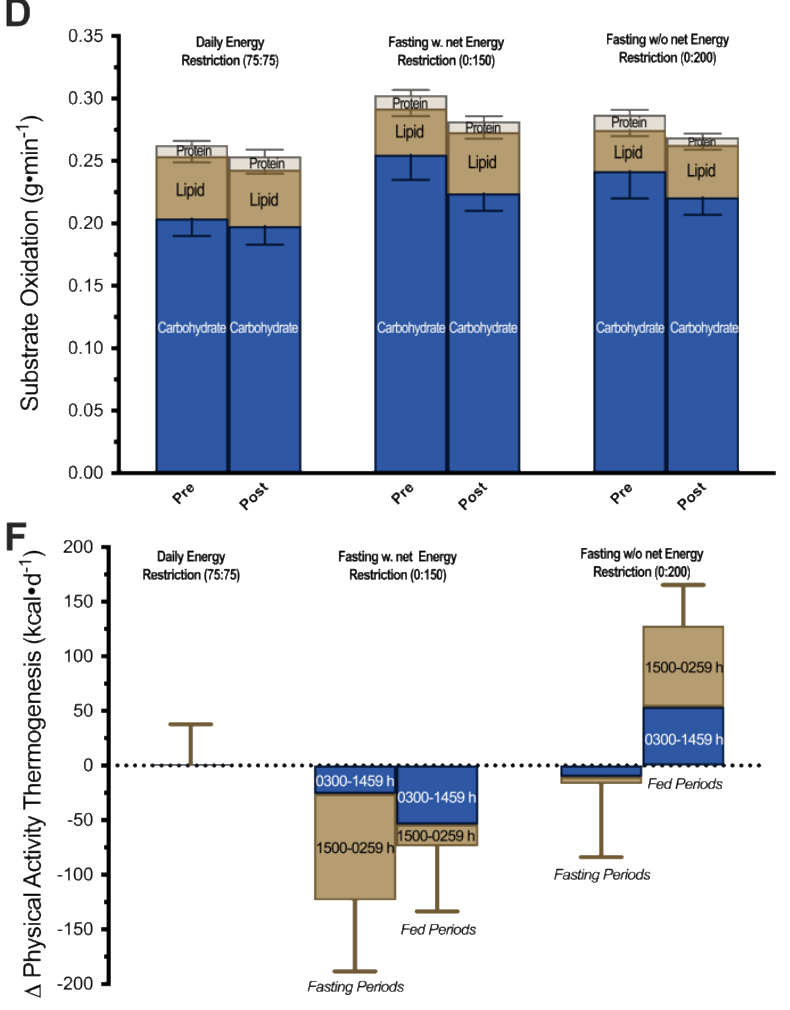
Science
Translational

Medicine Submitted Manuscript: Confidential

template updated: February 282012

MAAS
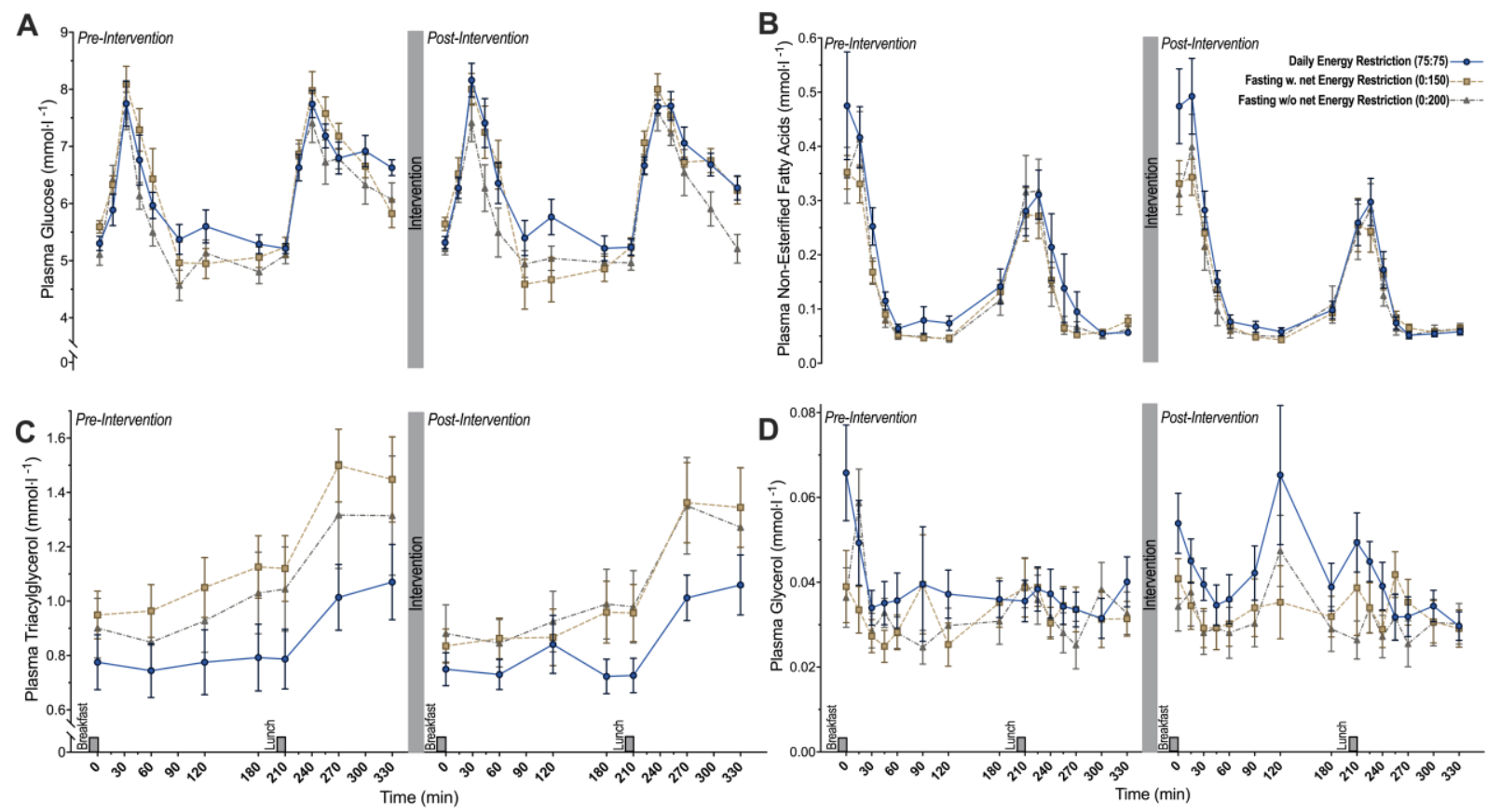

Figure 2
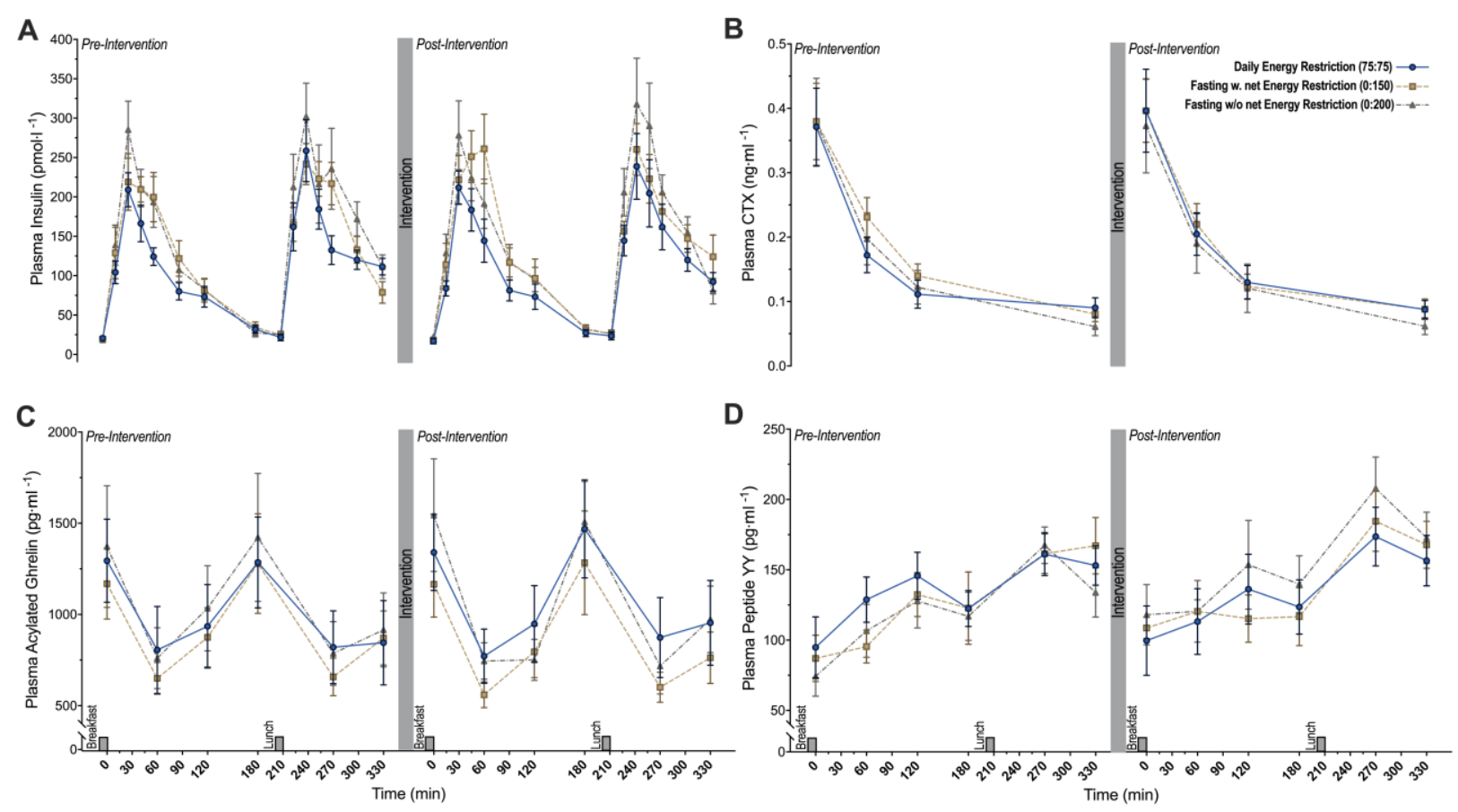

Figure 3 

DIAAAS

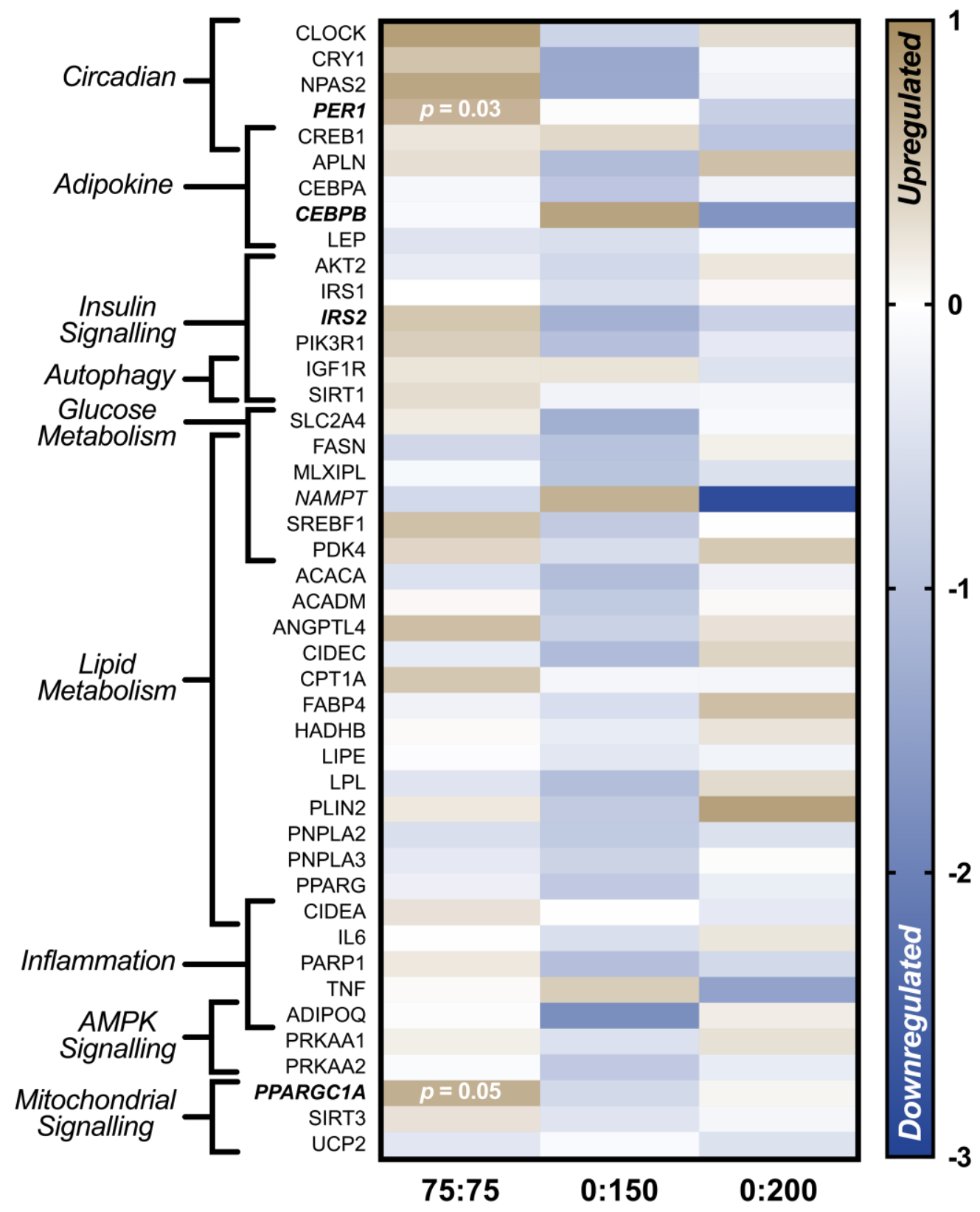

Figure 4 